Optimizing the electron-withdrawing character on benzene sulfonyl moiety attached to a glyco-conjugate to impart sensitive and selective sensing of cyanide in HEPES buffer and on cellulose paper and silica gel strips

Sivaiah Areti, Sateesh Bandaru, Deepthi S. Yarramala and Chebrolu Pulla Rao* Bioinorganic Laboratory, Department of Chemistry, Indian Institute of Technology Bombay, Powai, Mumbai - 400076, India, E-mail: cprao@iitb.ac.in

\title{
Contents
}

Page no:

S01. Synthesis and characterization of $\mathbf{P}_{2}$

S02. Synthesis and characterization of $\mathbf{P}_{4}$

S03. Synthesis and characterization of $\mathbf{P}_{5}$

S04. Synthesis and characterization of ${ }^{5 \mathrm{~F}} \mathbf{L}_{\mathbf{O A c}}$

S05. Synthesis and characterization of ${ }^{2 \mathrm{~F}} \mathbf{L}_{\mathbf{O A c}}$

S06. Synthesis and characterization of ${ }^{1 \mathrm{~F}} \mathbf{L}_{\mathbf{O A c}}$

S07. Synthesis and characterization of ${ }^{0 \mathrm{~F}} \mathbf{L}_{\mathbf{O A c}}$

S08. Synthesis and Characterization of ${ }^{5 \mathrm{~F}} \mathbf{L}_{\mathbf{O H}}$

S09. Synthesis and Characterization of ${ }^{2 \mathrm{~F}} \mathbf{L}_{\mathbf{O H}}$

S10. Synthesis and Characterization of ${ }^{1 \mathrm{~F}} \mathbf{L}_{\mathbf{O H}}$

S11. Synthesis and Characterization of ${ }^{0 \mathrm{~F}} \mathbf{L}_{\mathbf{O H}}$

S12. Characterization of $\mathbf{L}_{1}$

S13. Crystallographic data for ${ }^{55} \mathbf{L}_{\text {OAc }}$

S14. pH Optimization for the reaction of ${ }^{5 \mathrm{~F}} \mathbf{L}_{\mathbf{O H}}$ with $\mathrm{CN}^{-}$

S15. Fluorescence studies of ${ }^{5 \mathrm{~F}} \mathbf{L}_{\mathbf{O H}}$ with anions and metal ions 25

S16. Determination of Limit of Detection (LOD) of $\mathrm{CN}^{-}$by ${ }^{5 \mathbf{F}} \mathbf{L}_{\mathbf{O H}}$

S17. Comparison of the detection limits of recently developed fluorescent probes for $\mathrm{CN}^{-}$in the literature.

S18. Absorption titration of ${ }^{5 \mathrm{~F}} \mathbf{L}_{\mathbf{O H}}$ with anions 28

S19. ESI MS spectrum for the titration of $\left[{ }^{5 \mathrm{~F}} \mathbf{L}_{\mathbf{O H}}\right]$ with $\mathrm{CN}^{-}$

S20. Determination of Limit of Detection (LOD) of $\mathrm{CN}^{-}$by ${ }^{5 \mathrm{~F}} \mathbf{L}_{\mathbf{O H}}$ on silica gel and paper strips

S21. Isothermal titration calorimetry study of ${ }^{\mathbf{n F}} \mathbf{L}_{\mathbf{O H}}$ with $\mathrm{CN}^{-}$ 
S01. Synthesis and characterization of $\mathbf{P}_{2}: \quad \mathbf{P}_{\mathbf{2}}$ derivative was synthesized by using a literature reported procedure. ${ }^{[R e f 1]}$ [Ref1]: (a) Bolletta, F.; Fabbri, D.; Lombardo, M.; Prodi, L.; Trombini, C.; Zaccheroni, N. Organometallics 1996, 15, 2415-2417.; (b) Drillaud, N.; Estelle, B.-L.; Pezron, I.; Len, C.| J. Org. Chem. 2012, 77, 9553-9561.

$\mathbf{P}_{2}$ was obtained as a yellow solid (85 \%). ${ }^{\mathbf{1}} \mathbf{H N M R}\left(\mathbf{4 0 0} \mathbf{M H z}, \mathbf{C D C l}_{\mathbf{3}}, \boldsymbol{\delta} \mathbf{~ p p m}\right): 1.9$ (t, $\mathrm{J}=2.2 \mathrm{~Hz}, 1 \mathrm{H}), 2.8(\mathrm{~s}, 6 \mathrm{H}), 3.8(\mathrm{~d}, \mathrm{~J}=7.4,2 \mathrm{H}), 4.62(\mathrm{t}, \mathrm{J}=14.8 \mathrm{~Hz}, 1 \mathrm{H}), 7.18(\mathrm{~d}, \mathrm{~J}=7.8 \mathrm{~Hz}$ 1H), 7.50-7.58 (m, 2H), 8.23-8.29 (m, 2H), $8.53(\mathrm{~d}, \mathrm{~J}=8.4 \mathrm{~Hz}, 1 \mathrm{H})$.

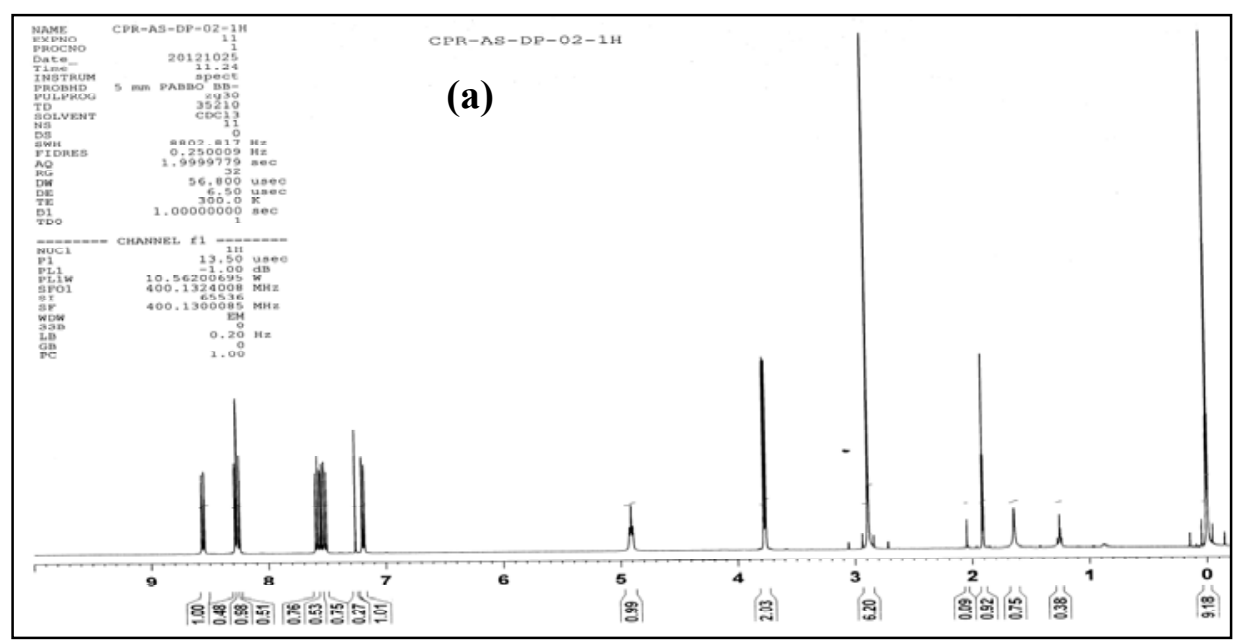

Figure S01: (a) ${ }^{1} \mathrm{H}$ NMR spectrum $\left(\mathrm{CDCl}_{3}, 400 \mathrm{MHz}\right)$ of $\mathbf{P}_{2}$.

S02. Synthesis and characterization of $\mathbf{P}_{\mathbf{4}}$ : This was synthesized by using a literature reported procedure. $^{[\operatorname{Ref2}]}$ [Ref2]: Nicolas, D.; Estelle, B.-L.; Isabelle, P.; Christophe, L. J. Org. Chem., 2012, 77, 9553-9561.

The yield is $69 \%$ as white crystals. ${ }^{1} \mathbf{H N M R}\left(\mathbf{4 0 0} \mathbf{~ M H z}, \mathbf{C D C l}_{3}, \boldsymbol{\delta} \mathbf{~ p p m}\right): 2.0(\mathrm{~s}, 3 \mathrm{H})$, $2.08(\mathrm{~s}, 3 \mathrm{H}), 2.12$ (s, 3H), 2.16 (s, 3H), 3.8-3.9 (m, 1H), 4.14 (d, $J=6.4 \mathrm{~Hz}, 1 \mathrm{H}), 4.28-4.3$ $(\mathrm{dd}, J=2.6,2.2 \mathrm{~Hz}, 1 \mathrm{H}), 4.65(\mathrm{~d}, J=7.6 \mathrm{~Hz}, 1 \mathrm{H}), 4.95$ (t, $J=13.2 \mathrm{~Hz}, 1 \mathrm{H}), 5.15(\mathrm{t}, J=13.8$ $\mathrm{Hz}, 1 \mathrm{H}) 5.25(\mathrm{t}, J=14.2 \mathrm{~Hz}, 1 \mathrm{H})$. 


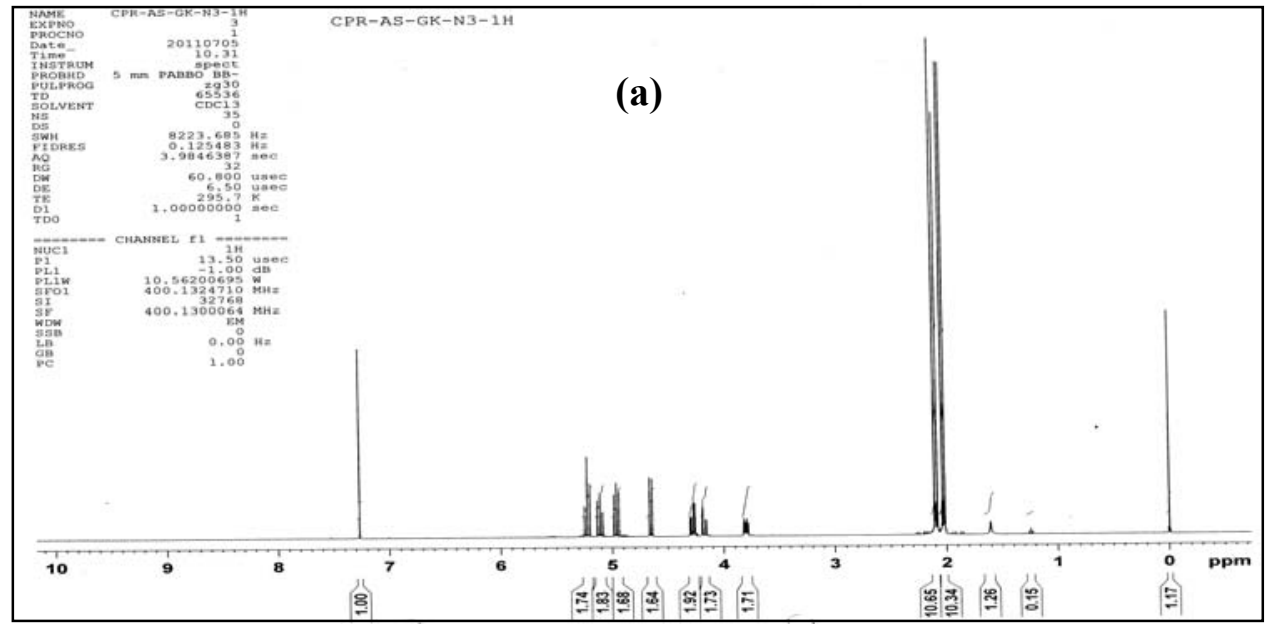

Figure S02. (a) ${ }^{1} \mathrm{H} \mathrm{NMR}\left(\mathrm{CDCl}_{3}, 400 \mathrm{MHz}\right)$ for $\mathbf{P}_{4}$.

S03. Synthesis and characterization of $\mathbf{P}_{\mathbf{5}}$ : A $0.37 \mathrm{~g}(0.1 \mathrm{mmol})$ of $\mathbf{P}_{\mathbf{4}}$ and $0.24 \mathrm{~g}(0.75$ mmol) of $\mathbf{P}_{2}$ were mixed in a $50 \mathrm{~mL}$ flask containing $12 \mathrm{~mL}$ of $1: 1 \mathrm{vol} / \mathrm{vol}$ ratio of tertiary-butanol : water. Then $0.06 \mathrm{~g}(0.4 \mathrm{mmol})$ of $\mathrm{CuSO}_{4} .5 \mathrm{H}_{2} \mathrm{O}$ and $0.022 \mathrm{~g}(0.8 \mathrm{mmol})$ of sodium ascorbate were added to the reaction medium. The mixture was allowed to stir magnetically at room temperature for $12 \mathrm{hrs}$. After the completion of the reaction, it was extracted with ethylacetate. The organic layer was then washed with water $(2 \times 20 \mathrm{~mL})$ and dried overnight over anhydrous $\mathrm{Na}_{2} \mathrm{SO}_{4}$. The organic layer was evaporated under vacuum and purified by column chromatography (silica gel) using ethyl acetate and pet ether mixture (2:3 vol/vol) as eluent, $\mathbf{P}_{5}$ was obtained as a solid in $72 \%$ yield. ${ }^{1} \mathbf{H}$ NMR (400 MHz, $\mathbf{C D C l}_{3}, \boldsymbol{\delta}$ ppm): 1.95 (S, 3H), $1.97(\mathrm{~S}, 3 \mathrm{H}), 2.0(\mathrm{~S}, 3 \mathrm{H}), 2.03(\mathrm{~S}, 3 \mathrm{H}), \delta 2.86$ (S. $6 \mathrm{H}) ; \delta 3.91-3.95(\mathrm{~m}, 1 \mathrm{H}) ; 4.10-4.14(\mathrm{~m}, 2 \mathrm{H}), 4.23(\mathrm{dd}, 1 \mathrm{H}, J=5.4, J=4.8 \mathrm{~Hz}), 5.16(\mathrm{t}$, $1 \mathrm{H}, J=14.2 \mathrm{~Hz}), 5.26-5.36(\mathrm{~m}, 2 \mathrm{H}), 5.49$ (t, $J=9.8 \mathrm{~Hz}, 1 \mathrm{H}), 5.73(\mathrm{~d}, J=8.9 \mathrm{~Hz}, 1 \mathrm{H}), 7.16$ (d, $J=7.48 \mathrm{~Hz}, 1 \mathrm{H}), 7.45-7.53(\mathrm{~m}, 2 \mathrm{H}), 7.63(\mathrm{~S}, 1 \mathrm{H}), 8.19-8.21$ (dd, $1 \mathrm{H}, J=4.2, J=4.4$ $\mathrm{Hz}, 1 \mathrm{H}) ; 8.25(\mathrm{~d}, J=8.64 \mathrm{~Hz}, 1 \mathrm{H}), 8.35$ (d, $J=8.04 \mathrm{~Hz}, 1 \mathrm{H}) .{ }^{\mathbf{1 3}} \mathbf{C}$ NMR (100 MHz, $\mathbf{C D C l}_{3}, \boldsymbol{\delta}$ ppm): 20.24, 20.63, 20.66, 20.81, 38.76, 45.55,61.65, 67.72, 70.45, 72.69, $75.11,85.71,115.46,119.0,121.29,123.36,128.64,129.65,129.75,129.89,130.75$, 134.49, 144.83, 169.04, 169.51, 170.08, 170.70. FTIR (KBr, $\left.\mathbf{~ c m}^{-\mathbf{1}}\right) ; 1235\left(v_{-0}\right), 1348$ $\left(v_{-\mathrm{S}}=\mathrm{O}\right), 1762\left(v_{-\mathrm{C}=\mathrm{O}}\right) 2937\left(v_{\mathrm{Ar}-\mathrm{C}-\mathrm{H}}\right)$ and $3341\left(v_{-\mathrm{N}-\mathrm{H}}\right)$. HRMS: Chemical Formula: $\mathrm{C}_{29} \mathrm{H}_{36} \mathrm{~N}_{5} \mathrm{O}_{11} \mathrm{~S}\left[\mathrm{M}+\mathrm{H}^{+}\right]$, calculated mass: 662.2132, observed mass 662.2177. 


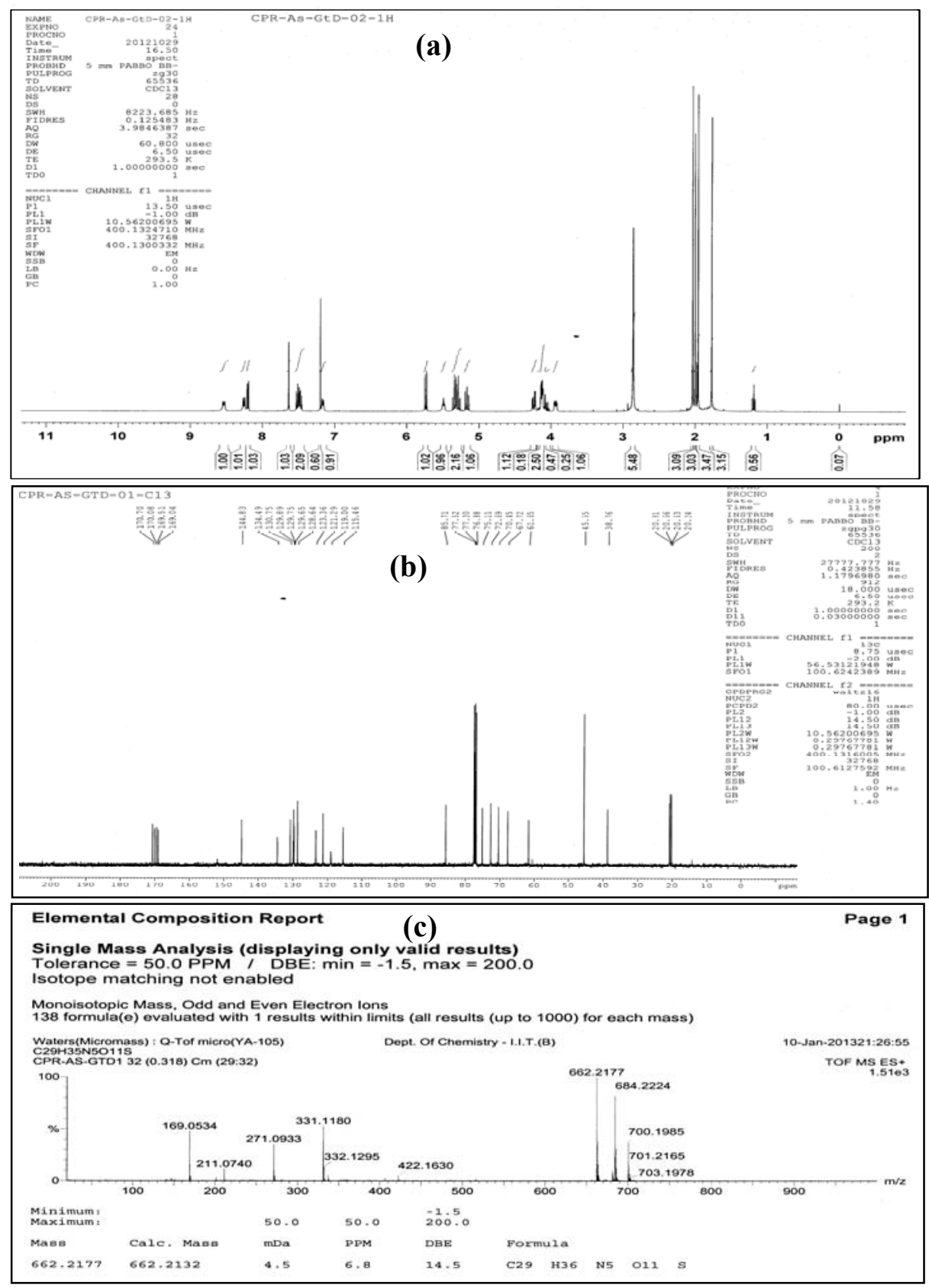

Figure S03 (a) ${ }^{1} \mathrm{H}$ NMR $\left(\mathrm{CDCl}_{3}, 400 \mathrm{MHz}\right)\left(\right.$ b) ${ }^{13} \mathrm{C} \mathrm{NMR}\left(\mathrm{CDCl}_{3}, 400 \mathrm{MHz}\right)(\mathrm{c}) \mathrm{HRMS}$ for $\mathbf{P}_{5}$. 
S04. Synthesis and characterization of ${ }^{5 \mathbf{F}} \mathbf{L}_{\mathbf{O A c}}$ : To a solution of $\mathbf{P}_{\mathbf{5}}(620 \mathrm{mg}, 10.8$ $\mathrm{mmol})$ and pentafluorobenzenesulfonyl chloride (PFBSCl) $(270.6 \mathrm{mg} 10.6 \mathrm{mmol})$ in anhydrous THF $(10 \mathrm{~mL}), \mathrm{NaH}(0.04 \mathrm{~g}, 0.15 \mathrm{mmol})$ was added at $0{ }^{\circ} \mathrm{C}$. The reaction mixture was warmed up to room temperature and stirred for another $1 \mathrm{hr}$ and was quenched with saturated $\mathrm{NaHCO}_{3}$ and the product was extracted using ethyl acetate. The combined organic layers were washed with brine, dried with sodium sulfate and then filtered and the solvent was evaporated under vacuum. The crude product was purified by silica gel chromatography with acetyl acetate and petether (4:6 vol/vol) which afforded desired product as green solid (80\% Yield). ${ }^{1} \mathbf{H}$ NMR (400 $\mathbf{M H z}, \mathbf{C D C l}_{3}, \boldsymbol{\delta}$ ppm): $2.0(\mathrm{~s}, 3 \mathrm{H}), 2.04(\mathrm{~s}, 3 \mathrm{H}), 2.08(\mathrm{~s}, 3 \mathrm{H}), 2.16(\mathrm{~s}, 3 \mathrm{H}), 2.8(\mathrm{~s}, 6 \mathrm{H}), 3.9-4.0(\mathrm{~m}, 1 \mathrm{H})$, $4.15(\mathrm{~d}, J=6.4 \mathrm{~Hz}, 1 \mathrm{H}), 4.26-4.3(\mathrm{dd}, J=3.4,2.8 \mathrm{~Hz}, 1 \mathrm{H}), 5.24$ (s, 2H), 5.4-5.44 (m, 2H), $5.84(\mathrm{~d}, J=6.4 \mathrm{~Hz}, 1 \mathrm{H}), 7.15(\mathrm{~d}, J=7.4 \mathrm{~Hz}, 1 \mathrm{H}), 7.5-7.6(\mathrm{~m}, 2 \mathrm{H}), 7.9(\mathrm{~s}, 1 \mathrm{H}), 8.05(\mathrm{~d}, J=$ $7.6 \mathrm{~Hz}, 1 \mathrm{H}), 8.42(\mathrm{~d}, J=7.8 \mathrm{~Hz}, 1 \mathrm{H}), 8.54(\mathrm{~d}, J=8.68 \mathrm{~Hz}, 1 \mathrm{H}) . \quad\left(\mathbf{1 0 0} \mathbf{~ M H z}, \mathbf{C D C l}_{3}, \boldsymbol{\delta}\right.$ ppm): 20.2, 20.6, 20.6, 20.8, 310., 48.9, 57.4, 61.5, 67.7, 70.5, 72.4, 75.2, 85.9, 103.6, $121.9,122.3,123.4,123.9,125.4,137.9,143.0,144.8,145.1,149.4,154.7,168.9,169.9$, 169.9, 170.5. FTIR $\left(\mathrm{KBr}, \mathbf{c m}^{-1}\right) ; 1232\left(v_{-\mathrm{O}-}\right), 1352\left(v_{-\mathrm{S}=\mathrm{O}}\right), 1760\left(v_{-\mathrm{C}=\mathrm{O}}\right)$ and $2937\left(v_{\text {Ar-C- }}\right.$ H). HRMS: Chemical Formula: $\mathrm{C}_{35} \mathrm{H}_{34} \mathrm{~F}_{5} \mathrm{~N}_{5} \mathrm{O}_{13} \mathrm{~S}_{2}\left[\mathrm{M}+\mathrm{H}^{+}\right]$, calculated mass: 914.1407: Observed mass: 914.1404.

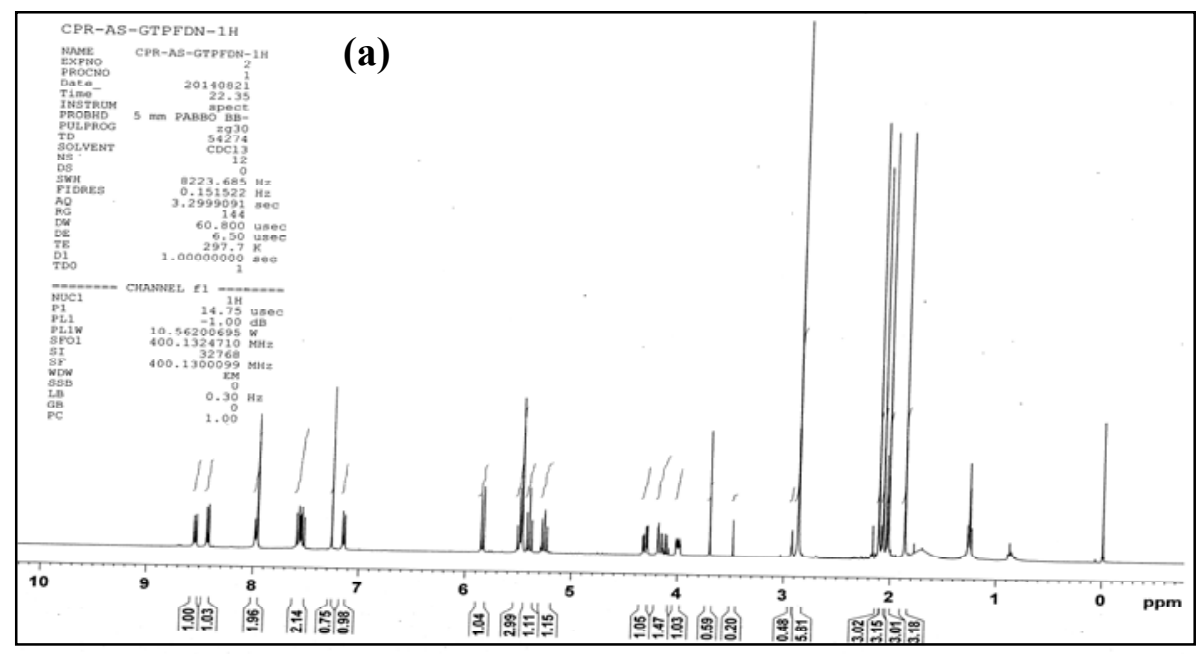



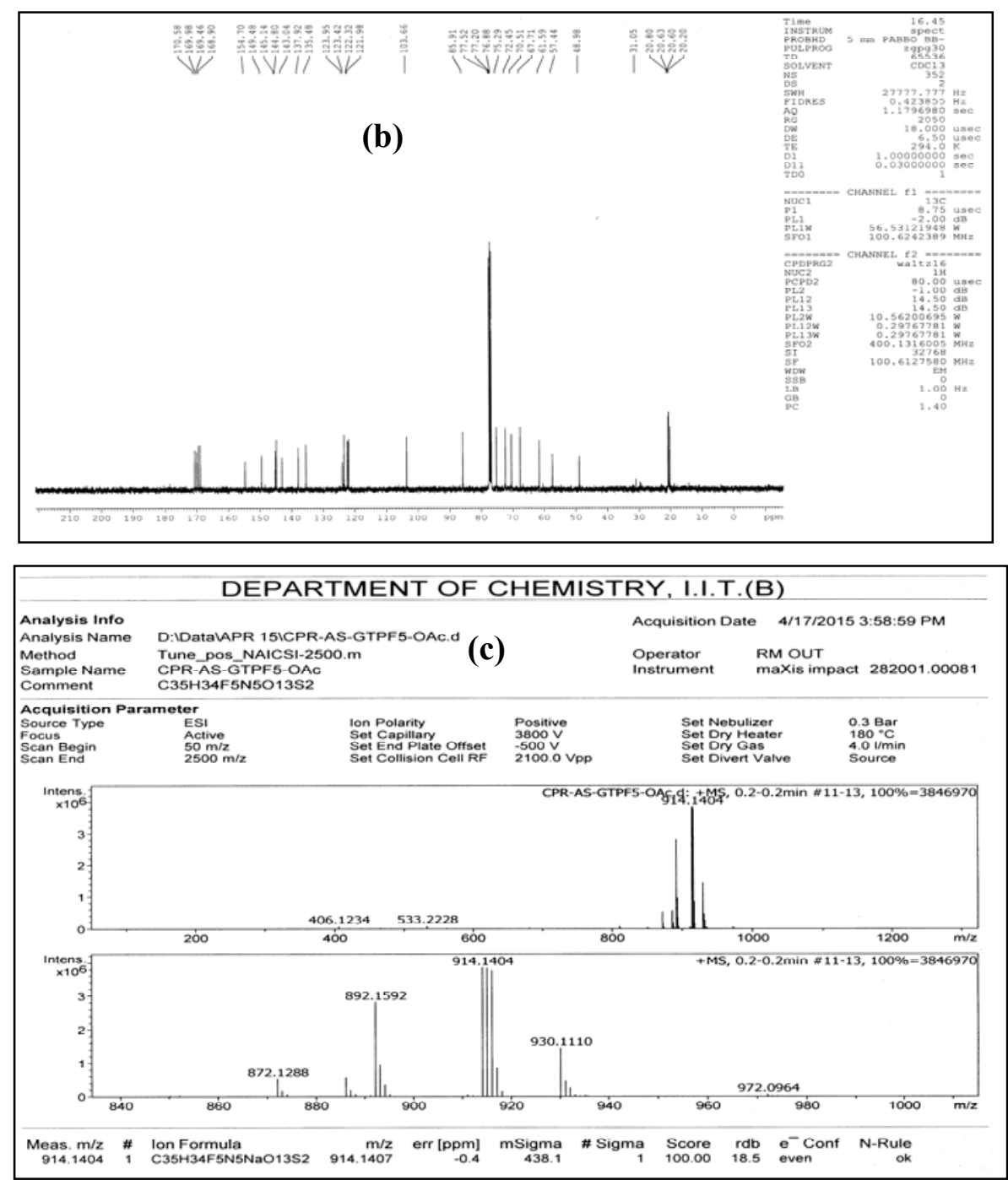

Figure S04. (a) ${ }^{1} \mathrm{H} \mathrm{NMR}\left(\mathrm{CDCl}_{3}, 400 \mathrm{MHz}\right)$ (b) ${ }^{13} \mathrm{C} \mathrm{NMR}\left(\mathrm{CDCl}_{3}, 400 \mathrm{MHz}\right)$ (c) HRMS for ${ }^{5 \mathbf{F}} \mathbf{L}_{\mathbf{O A c}}$.

S05. Synthesis and characterization of ${ }^{2 \mathrm{~F}} \mathbf{L}_{\mathrm{OAc}}$ : Same procedure has been followed as that of ${ }^{5 F} \mathbf{L}_{\mathbf{O A c}}$. $\mathbf{P}_{\mathbf{5}}(620 \mathrm{mg}, 10.8 \mathrm{mmol}), 2,4 \mathrm{DFBS}-\mathrm{Cl}(212.5 \mathrm{mg} 10.6 \mathrm{mmol})$ to obtain the product in $65 \%$ yield as greenish solid. ${ }^{1} \mathbf{H}$ NMR (400 $\left.\mathbf{M H z}, \mathrm{CDCl}_{3}, \boldsymbol{\delta} \mathbf{~ p p m}\right): 2.02$ (s, 3H), 2.03 (s, 3H), 2.04 (s, 3H), 2.07 (s, 3H), 2.85 (s, 6H), 2.90 (s, 1H), 3.95-3.99 (m, $1 \mathrm{H}), 4.09-4.14$ (q, 1H), 4.19-4.23 (dd, $J=2.8,2.4 \mathrm{~Hz}, 1 \mathrm{H}), 4.28-4.33$ (dd, $J=3.2,2.6 \mathrm{~Hz}$, $1 \mathrm{H}), 5.25(\mathrm{~s}, 2 \mathrm{H}), 5.39-5.41(\mathrm{~m}, 2 \mathrm{H}), 5.74(\mathrm{~d}, J=6.4 \mathrm{~Hz}, 1 \mathrm{H}), 7.11(\mathrm{~d}, J=7.4 \mathrm{~Hz}, 1 \mathrm{H})$, $7.41(\mathrm{t}, J=14.2 \mathrm{~Hz}, 1 \mathrm{H}), 7.57(\mathrm{t}, J=15.4 \mathrm{~Hz}, 1 \mathrm{H}), 7.83$ (s, 1H), 7.99 (d, $J=7.2 \mathrm{~Hz}, 1 \mathrm{H})$, 
$8.28(\mathrm{~d}, J=7.6 \mathrm{~Hz}, 1 \mathrm{H}), 8.32-8.36(\mathrm{~m}, 2 \mathrm{H}), 8.46(\mathrm{~d}, J=6.8 \mathrm{~Hz}, 1 \mathrm{H}), 8.59$ (d, $J=7.0 \mathrm{~Hz}$, 1H). ${ }^{13} \mathbf{C}$ NMR (100 MHz, $\mathbf{C D C l}_{3}, \boldsymbol{\delta}$ ppm): 20.19; 20.62; 20.66; 20.82; 44.69; 45.43; $60.51-85.68 ; 115.59,117.72,119.04,119,80,120.95,122.85,123.29,126.21,126.81$, $129.78,130.11,132.27,133.23,133.30,134.45,137.77,142.92,147.97,150.04,152.22$, 168.92, 169.43, 170.09, 170.68. ESI MS: Chemical Formula: $\mathrm{C}_{35} \mathrm{H}_{37} \mathrm{~F}_{2} \mathrm{~N}_{5} \mathrm{O}_{13} \mathrm{~S}_{2}\left[\mathrm{M}+\mathrm{K}^{+}\right]$, calculated mass 876.1429 , observed mass 876.1428 .
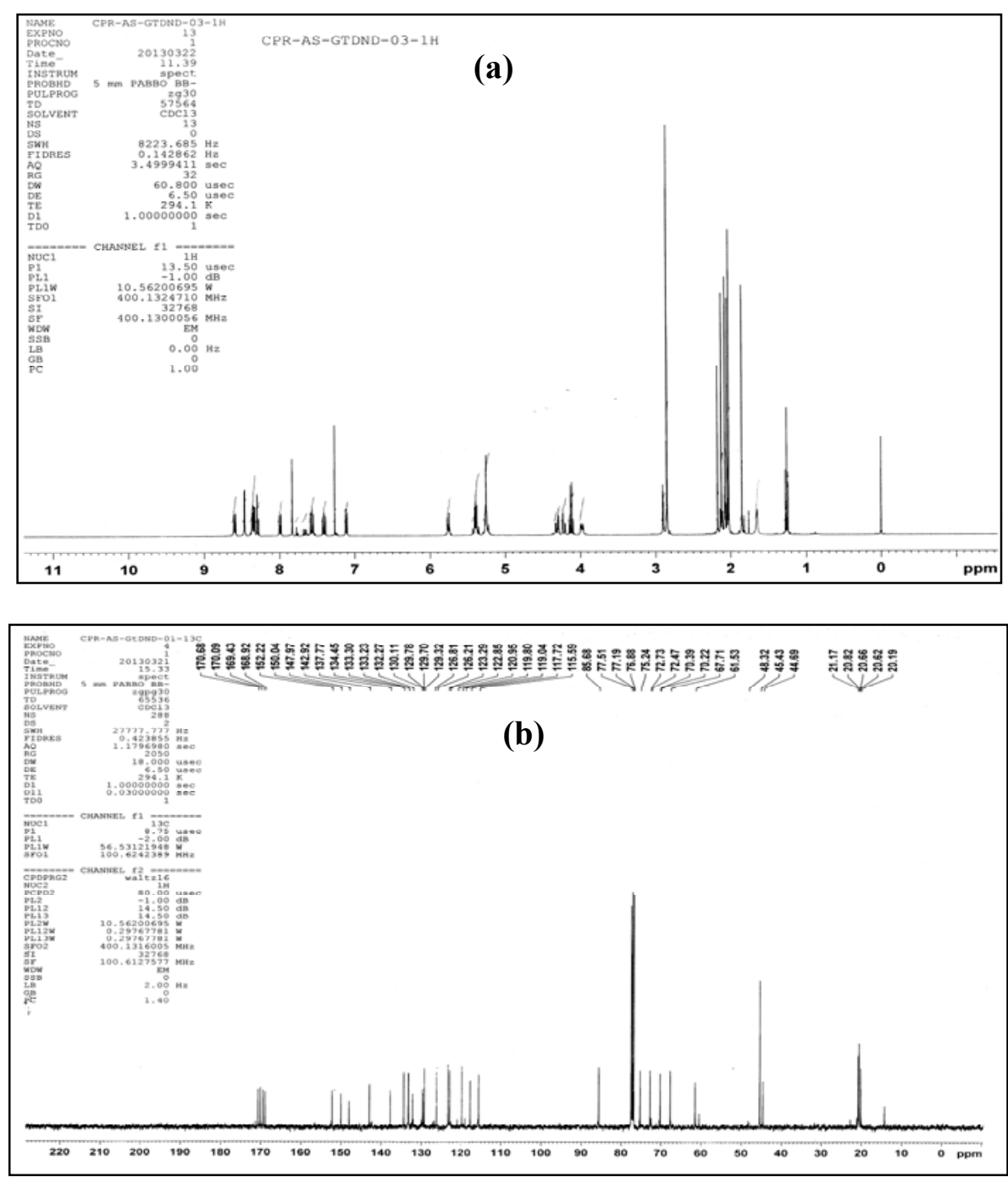


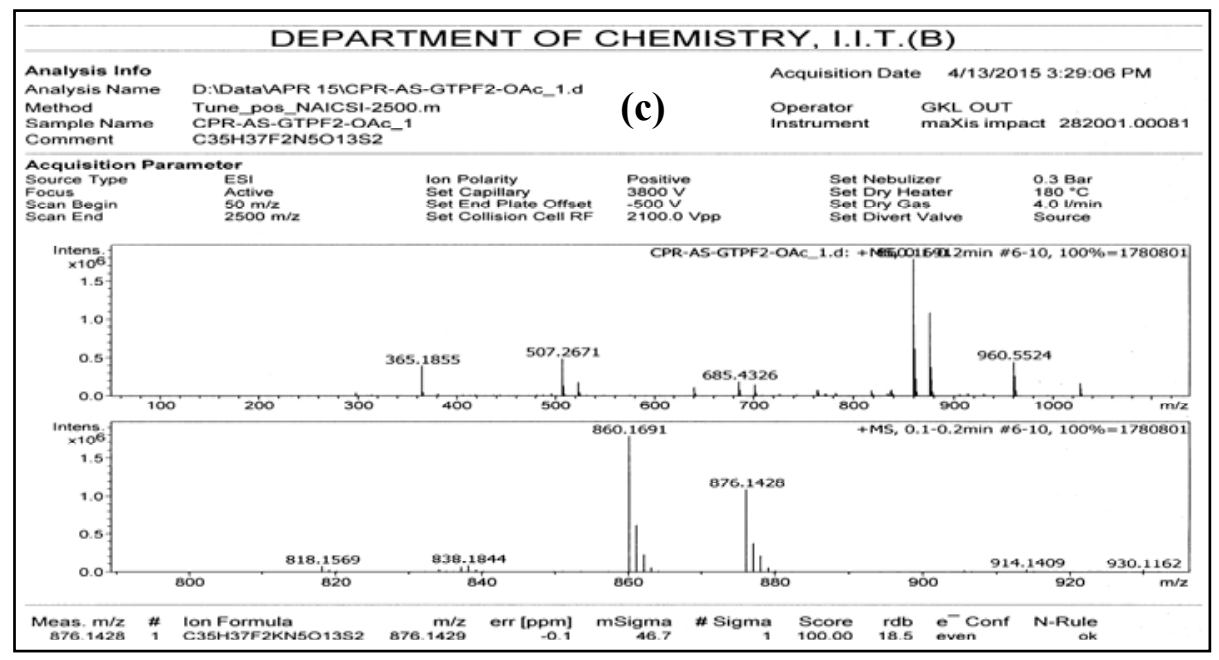

Figure S05. (a) ${ }^{1} \mathrm{H} \mathrm{NMR}\left(\mathrm{CDCl}_{3}, 400 \mathrm{MHz}\right)\left(\right.$ b) ${ }^{13} \mathrm{C} \mathrm{NMR}\left(\mathrm{CDCl}_{3}, 400 \mathrm{MHz}\right)$ (c) HRMS for ${ }^{2 \mathrm{~F}} \mathbf{L}_{\text {OAc. }}$

S06. Synthesis and characterization of ${ }^{1 \mathrm{~F}} \mathrm{~L}_{\mathrm{OAc}}$ : Same procedure has been followed as that of ${ }^{5 \mathbf{F}} \mathbf{L}_{\mathbf{O A c}} . \mathbf{P}_{\mathbf{5}}$ (620 mg, $10.8 \mathrm{mmol}$ ), 4-Fluoro benzene sulfonyl chloride (4-FBS-Cl) (212.5 mg $10.6 \mathrm{mmol}$ ) to obtain the product in $61 \%$ yield as greenish solid. ${ }^{\mathbf{1}} \mathbf{H}$ NMR (400 MHz, $\mathbf{C D C l}_{3}, \boldsymbol{\delta}$ ppm): 2.0 (s, 3H), $2.06(\mathrm{~s}, 3 \mathrm{H}), 2.12(\mathrm{~s}, 3 \mathrm{H}), 2.16(\mathrm{~s}, 3 \mathrm{H}), 2.89$ (s, $6 \mathrm{H}), 3.95-4.0(\mathrm{~m}, 1 \mathrm{H}), 4.05-4.15(\mathrm{~m}, 2 \mathrm{H}), 4.2(\mathrm{~d}, J=5.6 \mathrm{~Hz}, 1 \mathrm{H}), 4.28-4.32(\mathrm{dd}, J=2.4$, $2.8 \mathrm{~Hz}, 1 \mathrm{H}), 5.2(\mathrm{t}, J=8.4 \mathrm{~Hz}, 2 \mathrm{H}), 5.25(\mathrm{~s}, 2 \mathrm{H}), 5.38-5.42(\mathrm{~m}, 2 \mathrm{H}), 5.75(\mathrm{~d}, J=6.4 \mathrm{~Hz}$, 1H), $7.02(\mathrm{t}, J=9.2 \mathrm{~Hz}, 1 \mathrm{H}), 7.15(\mathrm{~d}, J=7.6 \mathrm{~Hz}, 1 \mathrm{H}), 7.50(\mathrm{t}, J=9.4 \mathrm{~Hz}, 1 \mathrm{H}), 7.62(\mathrm{~m}$, 1H), 7.7-7.8 (m, 2H), $7.85(\mathrm{~s}, 1 \mathrm{H}), 8.05(\mathrm{~d}, J=7.2 \mathrm{~Hz}, 1 \mathrm{H}), 8.41(\mathrm{~d}, J=7.8 \mathrm{~Hz}, 1 \mathrm{H}), 8.62$ $(\mathrm{d}, J=8.2 \mathrm{~Hz}, 1 \mathrm{H}) .{ }^{13} \mathbf{C} \mathbf{N M R}\left(\mathbf{1 0 0} \mathbf{~ M H z}, \mathbf{C D C l}_{3}, \boldsymbol{\delta} \mathbf{~ p p m}\right): 20.31,20.74,20.90,20.12$; $43.91,45.59,53.61,61.06,67.83,70.38,72.89,85.79,115.52,117.82,123.2,123.33$, $123.76,129.24,129.74,129.85,132.44,132.75,133.55,143.11,144.06,150.40,152.30$, 168.95, 169.47, 170.11, 170.69. HRMS: Chemical Formula: $\mathrm{C}_{35} \mathrm{H}_{38} \mathrm{FN}_{5} \mathrm{O}_{13} \mathrm{~S}_{2}\left[\mathrm{M}+\mathrm{Na}^{+}\right]$, calculated mass: 842.1784 , observed mass: 842.1783 . 

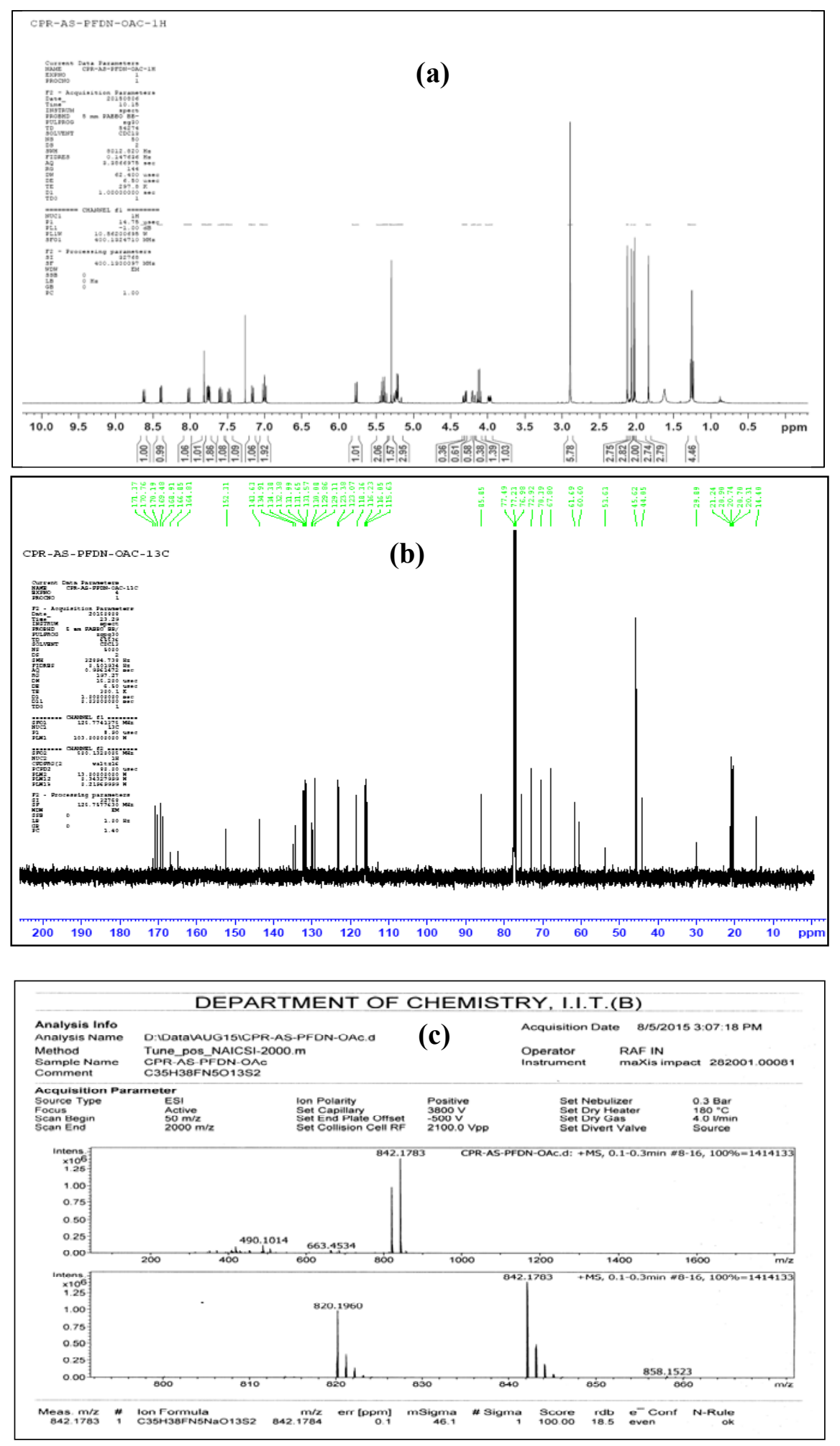

Figure S06. (a) ${ }^{1} \mathrm{H} \mathrm{NMR}\left(\mathrm{CDCl}_{3}, 400 \mathrm{MHz}\right)\left(\right.$ b) ${ }^{13} \mathrm{C} \mathrm{NMR}\left(\mathrm{CDCl}_{3}, 400 \mathrm{MHz}\right)(\mathrm{c})$ HRMS for ${ }^{1 \mathbf{F}} \mathbf{L}_{\text {OAc. }}$. 
S07. Synthesis and characterization of ${ }^{0 \mathrm{~F}} \mathrm{~L}_{\mathrm{OAc}}$ : Same procedure has been followed as that of ${ }^{\mathbf{5 F}} \mathbf{L}_{\mathbf{O A c}} . \quad \mathbf{P}_{\mathbf{5}}(620 \mathrm{mg}, 10.8 \mathrm{mmol})$, benzene sulfonyl chloride (BS-Cl) (215.5 $\mathrm{mg}$ $10.6 \mathrm{mmol})$ to obtain the product in $61 \%$ yield as greenish solid. ${ }^{1} \mathbf{H}$ NMR (400 MHz, $\mathbf{C D C l}_{3}, \boldsymbol{\delta}$ ppm): $2.0(\mathrm{~s}, 3 \mathrm{H}), 2.02(\mathrm{~s}, 3 \mathrm{H}), 2.10(\mathrm{~s}, 3 \mathrm{H}), 2.14(\mathrm{~s}, 3 \mathrm{H}), 2.89(\mathrm{~s}, 6 \mathrm{H}), 3.95-$ $4.0(\mathrm{~m}, 1 \mathrm{H}), 4.06-4.14(\mathrm{~m}, 2 \mathrm{H}), 4.2(\mathrm{~d}, J=5.8 \mathrm{~Hz}, 1 \mathrm{H}), 4.3-4.34(\mathrm{dd}, J=3.4,2.8 \mathrm{~Hz}, 1 \mathrm{H})$, $5.2(\mathrm{t}, J=9.4 \mathrm{~Hz}, 2 \mathrm{H}), 5.26(\mathrm{~s}, 2 \mathrm{H}), 5.38-5.44(\mathrm{~m}, 2 \mathrm{H}), 5.75(\mathrm{~d}, J=6.4 \mathrm{~Hz}, 1 \mathrm{H}), 7.1-7.15$ $(\mathrm{m}, 3 \mathrm{H}), 7.4(\mathrm{t}, J=9.8 \mathrm{~Hz}, 1 \mathrm{H}), 7.6-7.8(\mathrm{~m}, 3 \mathrm{H}), 7.85(\mathrm{~s}, 1 \mathrm{H}), 8.05(\mathrm{~d}, J=7.6 \mathrm{~Hz}, 1 \mathrm{H})$, $8.42(\mathrm{~d}, J=7.4 \mathrm{~Hz}, 1 \mathrm{H}), 8.6(\mathrm{~d}, J=7.8 \mathrm{~Hz}, 1 \mathrm{H}) .{ }^{13} \mathbf{C} \mathbf{N M R}\left(\mathbf{1 0 0} \mathbf{M H z}, \mathbf{C D C l}_{3}, \boldsymbol{\delta}\right.$ ppm):20.27, 20.71, 20.92, 21.86, 43.8, 45.62, 61.74, 67.86, 70.35, 72.92, 85.74, 115.36, $118.48,123.02,123.34,128.68,128.86,129.56,129.8,129.98,132.01,132.16,134.36$, 135.84, 144.0, 145.12, 152.1, 168.8, 169.42, 170.19 and 170.72. HRMS: Chemical Formula: $\mathrm{C}_{36} \quad \mathrm{H}_{41} \mathrm{~N}_{5} \mathrm{O}_{13} \mathrm{~S}_{2} \quad\left[\mathrm{M}+\mathrm{Na}^{+}\right]$, calculated mass: 838.2033, observed mass: 838.2034 .

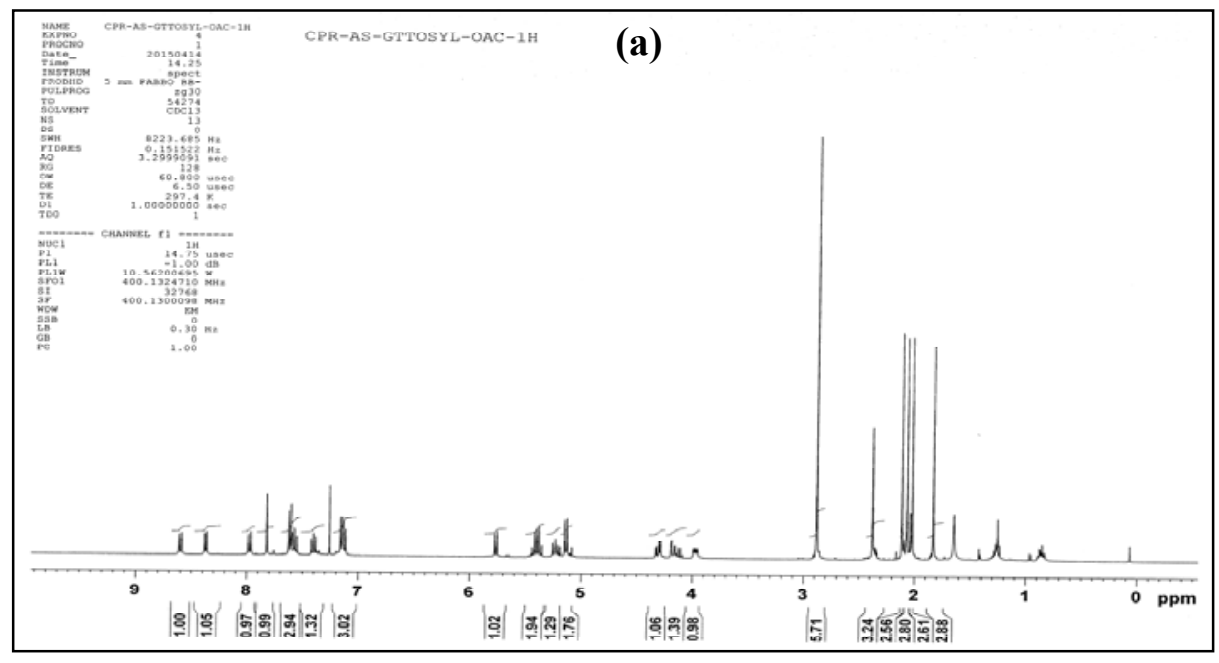



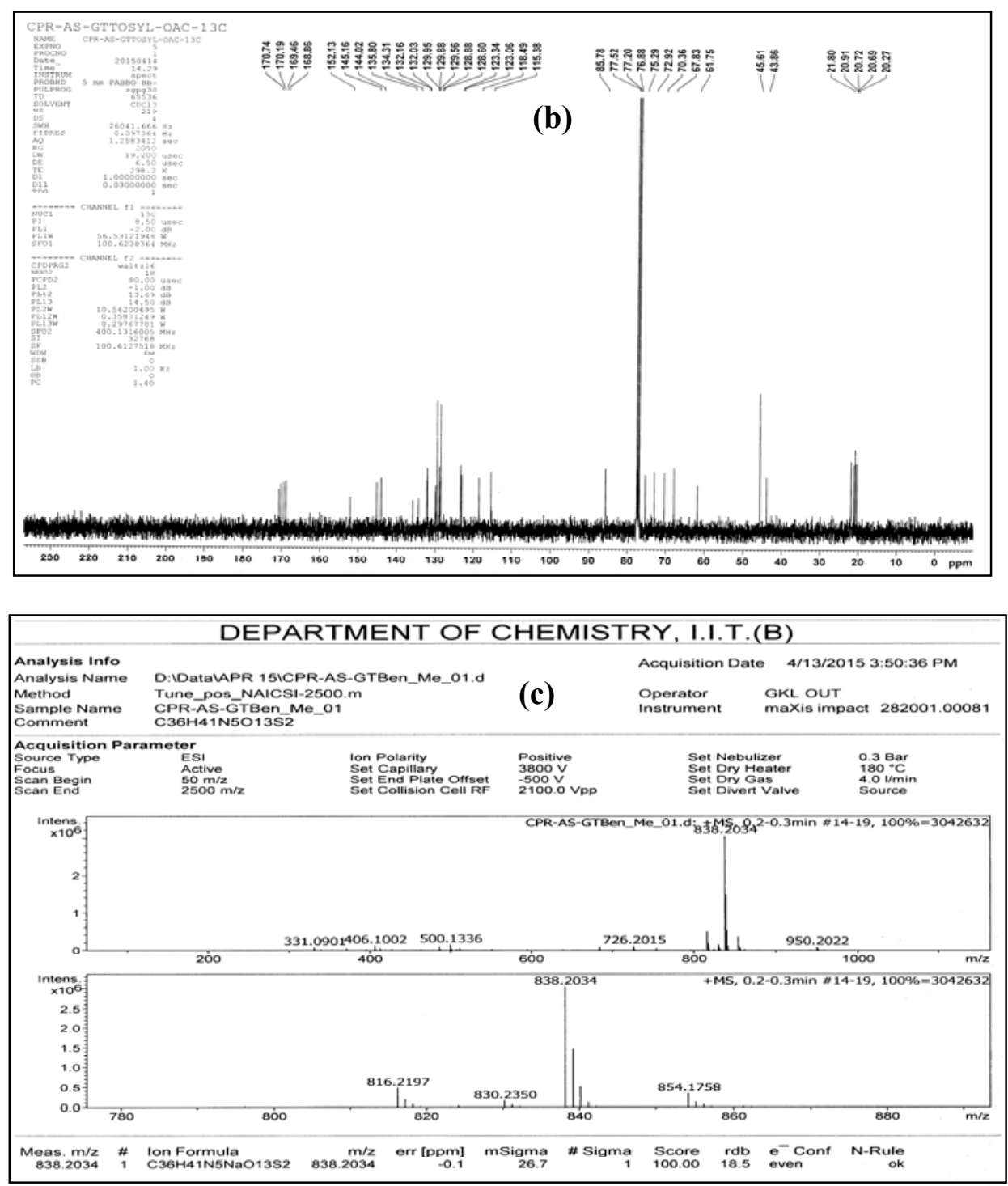

Figure S07: (a) ${ }^{1} \mathrm{H} \mathrm{NMR}\left(\mathrm{CDCl}_{3}, 400 \mathrm{MHz}\right)(\mathrm{b}){ }^{13} \mathrm{C} \mathrm{NMR}\left(\mathrm{CDCl}_{3}, 400 \mathrm{MHz}\right)(\mathrm{c}) \mathrm{HRMS}$ for ${ }^{0 \mathrm{~F}} \mathbf{L}_{\mathbf{O A c}}$. 


\section{S08. Synthesis and characterization of ${ }^{5 \mathrm{~F}} \mathrm{~L} \mathrm{OH}$}

To a solution of ${ }^{\mathbf{5 F}} \mathbf{L}_{\mathbf{O A c}}(890 \mathrm{mg}, 0.01 \mathrm{mmol})$ in $3 \mathrm{~mL}$ of $\mathrm{MeOH}$ was added drop wise acetyl chloride $(0.2 \mathrm{~mL}$ from a $0.02 \mathrm{M}$ solution in $\mathrm{MeOH})$. The reaction mixture was stirred at room temperature until the starting material was consumed as checked by TLC. The solution was neutralized by the addition of sodium bicarbonate and was filtered. The solvent was then removed in vacuum and obtained a solid product in $75 \%$ yield. ${ }^{\mathbf{1}} \mathbf{H}$ NMR (400 MHz, D $\mathbf{2}$, א ppm): 2.85 (s, 6H), 3.3 (s, 2H) 3.45-3.55 (m, 2H), 3.7-3.8 (m, 2H), $3.9(\mathrm{~d}, J=7.8 \mathrm{~Hz}, 1 \mathrm{H}), 4.15(\mathrm{~s}, 2 \mathrm{H}), 5.3(\mathrm{~d}, J=7.8 \mathrm{~Hz}, 1 \mathrm{H}), 7.28(\mathrm{~d}, J=8.6 \mathrm{~Hz}, 1 \mathrm{H})$, 7.56-7.50 (m, 3H), $7.85(\mathrm{~s}, 1 \mathrm{H}), 8.25(\mathrm{~d}, J=8.4 \mathrm{~Hz}, 1 \mathrm{H}), 8.35(\mathrm{~d}, J=7.6 \mathrm{~Hz}, 1 \mathrm{H}), 8.55(\mathrm{~d}$, $J=8.6 \mathrm{~Hz}, 1 \mathrm{H}) .{ }^{13} \mathbf{C}$ NMR (100 MHz, $\left.\mathbf{C D}_{3} \mathbf{O D}, \boldsymbol{\delta} \mathbf{p p m}\right): \quad 30.8,45.7,62.4,70.9,74.0$, $78.5,81.2,89.6,116.8,119.0,121.2,124.6,125.6,127.4,130.6,130.8,133.0,134.2$, 134.3, 137.7, 143.8, 149.0, 151.6, 153.4. FTIR (KBr, $\left.\mathbf{c m}^{-1}\right) ; 1344\left(v_{-\mathrm{S}=\mathrm{O}}\right), 2851\left(v_{-\mathrm{C}-\mathrm{H}}\right)$, $2923\left(v_{\text {Ar-C-H }}\right)$, and $3427\left(v_{-\mathrm{O}-\mathrm{H}}\right)$. HRMS: Chemical Formula is $\mathrm{C}_{27} \mathrm{H}_{26} \mathrm{~F}_{5} \mathrm{~N}_{5} \mathrm{O}_{9} \mathrm{~S}_{2}$ $\left[\mathrm{M}+\mathrm{Na}^{+}\right]$, Calculated mass is 746.0984, found is 746.0981. Anal. Calcd. for $\mathrm{C}_{27} \mathrm{H}_{26} \mathrm{~F}_{5} \mathrm{~N}_{5} \mathrm{O}_{9} \mathrm{~S}_{2} \cdot \mathrm{CH}_{3} \mathrm{COOCH}_{2} \mathrm{CH}_{3}$ : Calcd: $\mathrm{C} 45.87 \% ; \mathrm{H} 4.22 \% ; \mathrm{N} 8.63 \%$ and $\mathrm{S} 7.90$ \% Found C45.50\%; H $3.85 \%$; N $8.18 \%$ and S $7.67 \%$.

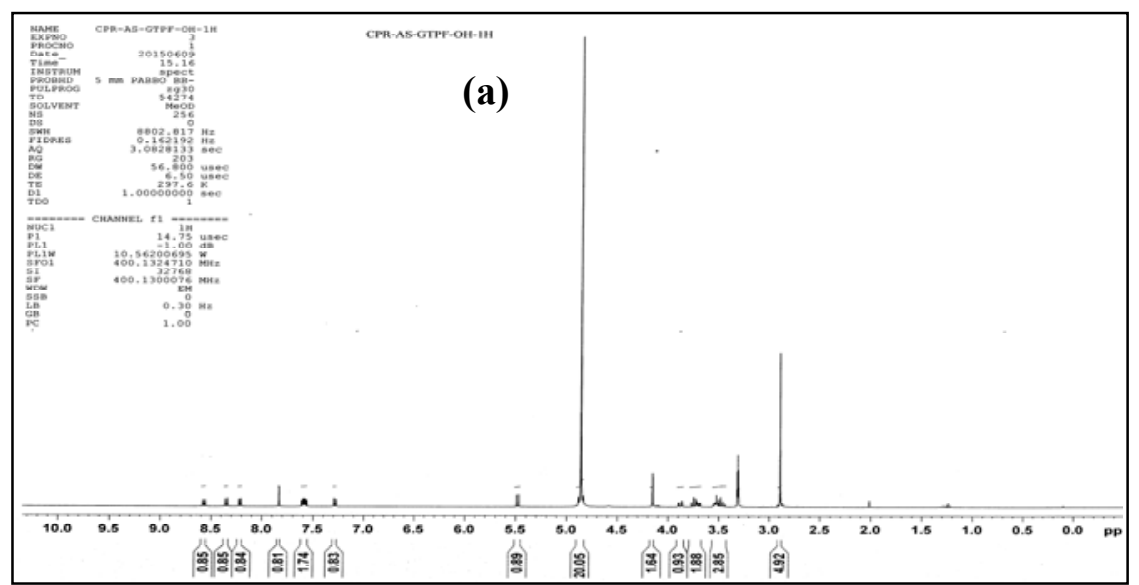



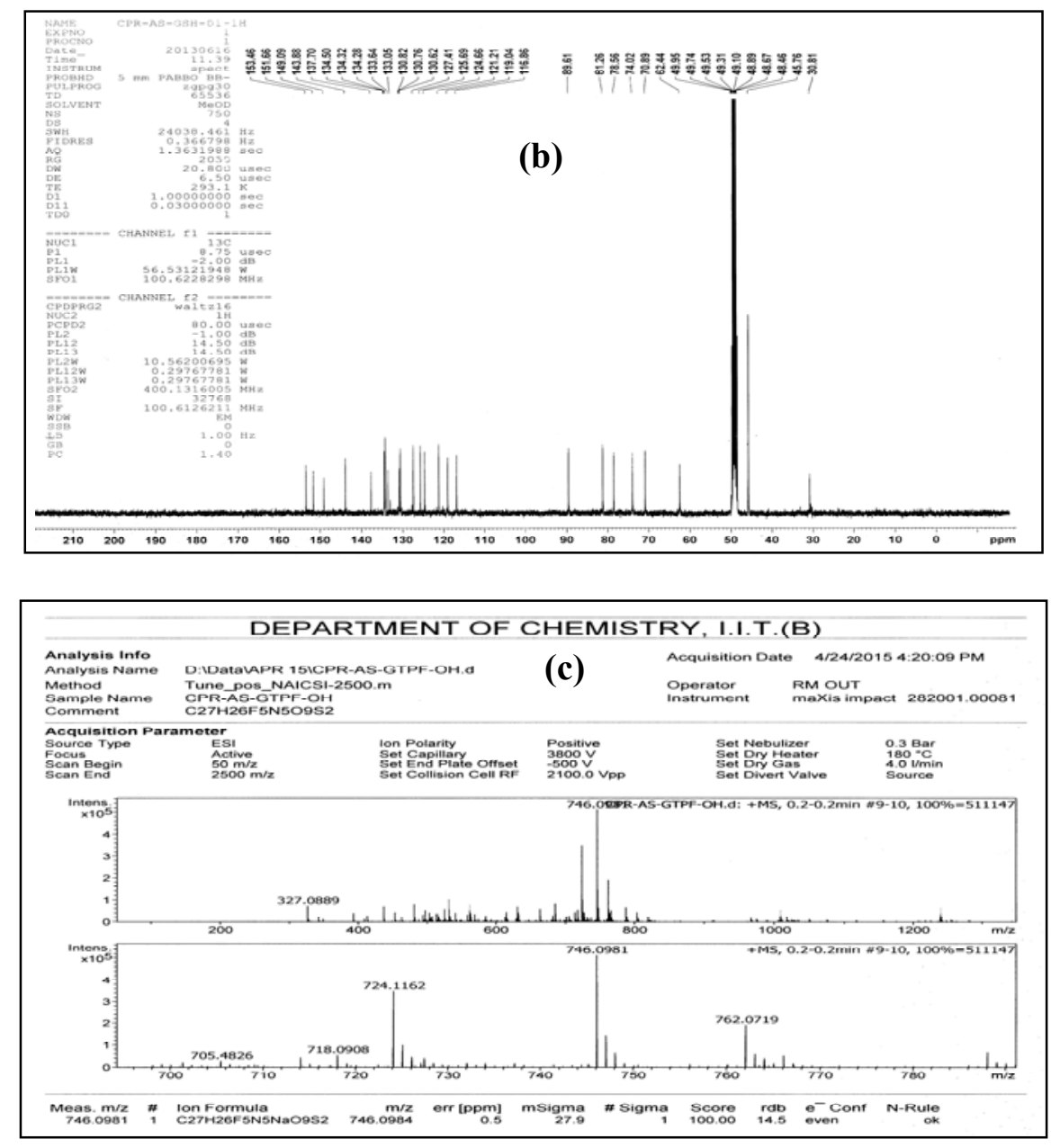

Figure S08: (a) ${ }^{1} \mathrm{H}$ NMR $\left(\mathrm{CD}_{3} \mathrm{OD}, 400 \mathrm{MHz}\right)(\mathrm{b}){ }^{13} \mathrm{C} \mathrm{NMR}\left(\mathrm{CD}_{3} \mathrm{OD}, 400 \mathrm{MHz}\right)(\mathrm{c}) \mathrm{HRMS}$ for ${ }^{5 \mathrm{~F}} \mathbf{L}_{\mathrm{OH}}$.

S09. Synthesis and Characterization of ${ }^{2 \mathrm{~F}} \mathbf{L}_{\mathrm{OH}}$ : The ${ }^{2 \mathrm{~F}} \mathbf{L}_{\mathrm{OH}}$ has been synthesized by adopting the procedure that was used for ${ }^{5 \mathbf{F}} \mathbf{L}_{\mathbf{O H}}$, but by using ${ }^{2 \mathbf{F}} \mathbf{L}_{\mathbf{O A c}}(860 \mathrm{mg}, 0.01 \mathrm{mmol})$ in place of ${ }^{5 \mathbf{F}} \mathbf{L}_{\mathbf{O A c}}$ in order to obtain the product in $75 \%$ yield as solid. ${ }^{1} \mathbf{H}$ NMR (400 MHz, CD $\mathbf{C}_{\mathbf{3}} \mathrm{OD}, \boldsymbol{\delta}$ ppm): 2.83 (S, 6H), 3.49-3.6 (m 2H),3.76 (dd, $\left.J=6.2 \mathrm{~Hz}, 1 \mathrm{H}\right), 3.84$ (t, $J=$ $13.8 \mathrm{~Hz}, 1 \mathrm{H}), 3.93(\mathrm{~d}, J=6.4 \mathrm{~Hz}, 1 \mathrm{H}), 5.37(\mathrm{~S}, 2 \mathrm{H}), 5.5(\mathrm{~d}, J=9.2 \mathrm{~Hz}, 1 \mathrm{H}), 7.19(\mathrm{~d}, J=7.8$ $\mathrm{Hz}, 1 \mathrm{H}), 7.49$ (t, $J=12.6 \mathrm{~Hz}, 1 \mathrm{H}), 7.64(\mathrm{t}, J=14.6 \mathrm{~Hz}, 1 \mathrm{H}), 8.01(\mathrm{t}, J=13.2 \mathrm{~Hz}, 2 \mathrm{H}), 8.12$ $(\mathrm{s}, 1 \mathrm{H}), 8.25(\mathrm{~d}, J=8.2 \mathrm{~Hz}, 1 \mathrm{H}), 8.33(\mathrm{~d}, J=8.6 \mathrm{~Hz}, 1 \mathrm{H}), 8.6(\mathrm{~m}, 2 \mathrm{H}) .{ }^{\mathbf{1 3}} \mathbf{C}$ NMR $(\mathbf{1 0 0}$ MHz, $\mathbf{C D}_{3} \mathrm{OD}, \boldsymbol{\delta}$ ppm): $30.81,45.76,62.44,70.89,74.02,78.56,81.26,89.61,116.86$, 
$119.04,121.21,124.66,125.69,127.41,130.62,130.76,130.82,133.64,134.28,134.32$, 134.50, 137.70, 143.88, 149.09, 151.66 and 153.46. FTIR (KBr, $\left.\mathbf{~ c m}^{-\mathbf{1}}\right) ; 1342\left(v_{-\mathrm{S}=0}\right), 2851$ $\left(v_{-\mathrm{C}-\mathrm{H}}\right), 2927\left(v_{\mathrm{Ar}-\mathrm{C}-\mathrm{H}}\right)$, and $3431\left(v_{-\mathrm{O}-\mathrm{H}}\right)$. HRMS: Chemical Formula is $\mathrm{C}_{27} \mathrm{H}_{28} \mathrm{~F}_{2} \mathrm{~N}_{5} \mathrm{O}_{9} \mathrm{~S}_{2}$ $\left[\mathrm{M}+\mathrm{H}^{+}\right]$, calculated mass is 668.1291 , found mass is 668.1290 .
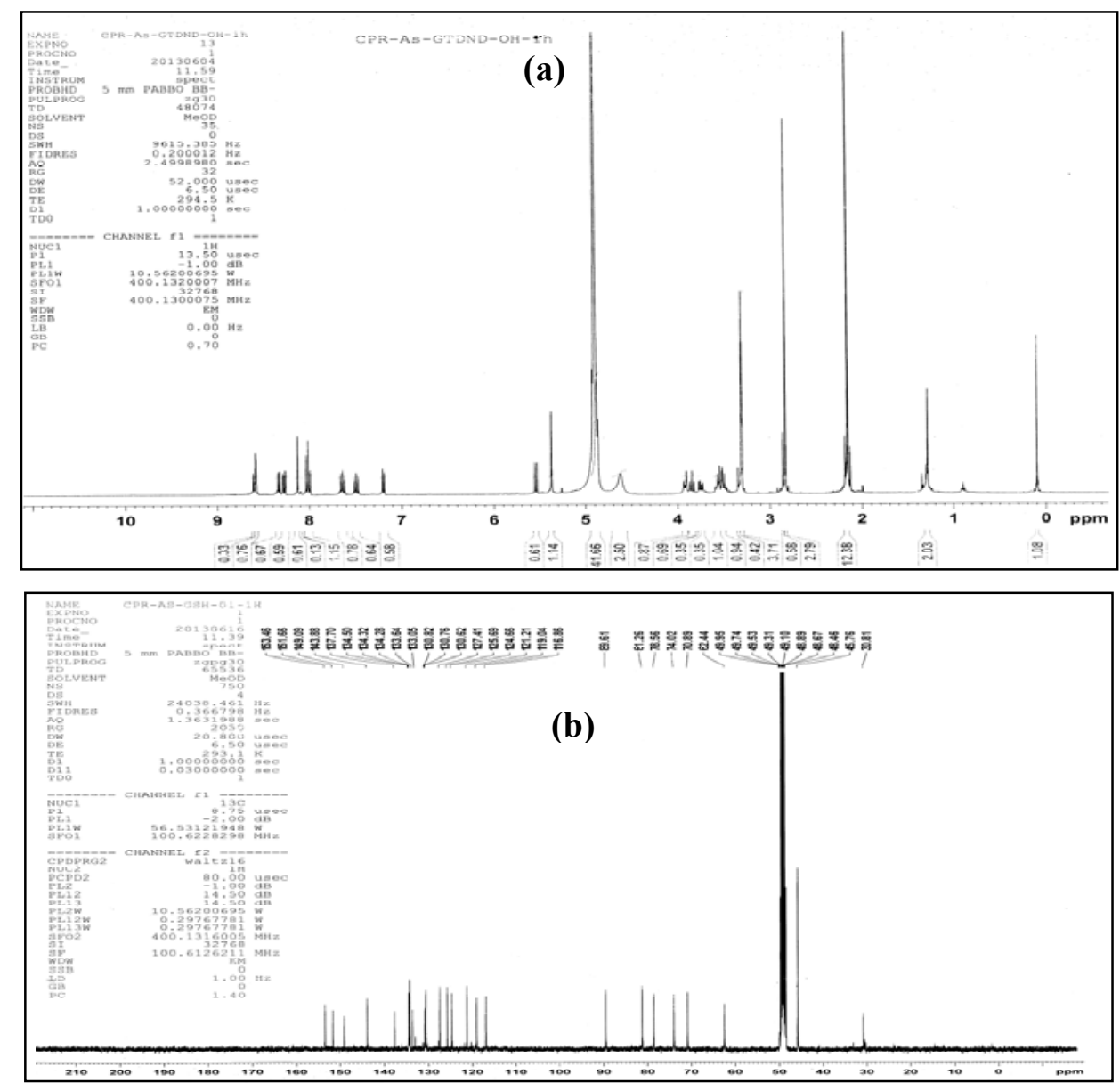


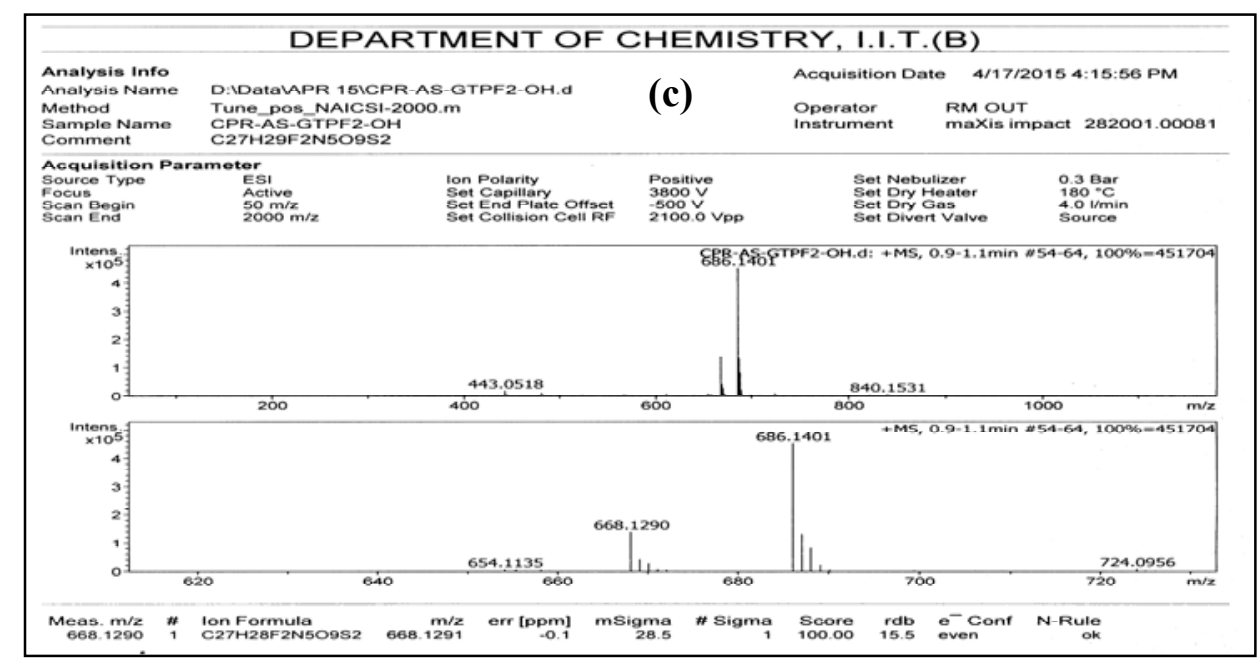

Figure S09: (a) ${ }^{1} \mathrm{H} \mathrm{NMR}\left(\mathrm{CDCl}_{3}, 400 \mathrm{MHz}\right)(\mathrm{b}){ }^{13} \mathrm{C} \mathrm{NMR}\left(\mathrm{CD}_{3} \mathrm{OD}, 400 \mathrm{MHz}\right)(\mathrm{c})$ HRMS for ${ }^{2 \mathbf{F}} \mathbf{L}_{\mathbf{O H}}$.

S10. Synthesis and Characterization of ${ }^{1 \mathrm{~F}} \mathbf{L}_{\mathrm{OH}}$ : The ${ }^{1 \mathrm{~F}} \mathbf{L}_{\mathrm{OH}}$ has been synthesized by adopting the procedure that was used for ${ }^{5 \mathbf{F}} \mathbf{L}_{\mathbf{O H}}$, but by using ${ }^{1 \mathrm{~F}} \mathbf{L}_{\mathbf{O A c}}(820 \mathrm{mg}, 0.01$ mmol) in place of ${ }^{\mathbf{5 F}} \mathbf{L}_{\mathbf{O A c}}$ in order to obtain the product in $68 \%$ yield as yellow solid. ${ }^{\mathbf{1}} \mathbf{H}$

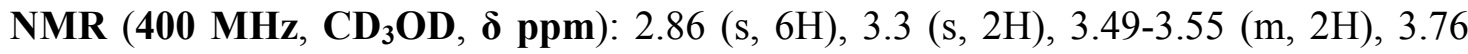
(dd, $J=4.2,3.8 \mathrm{~Hz}, 1 \mathrm{H}), 3.9$ (t, $J=8.4 \mathrm{~Hz}, 1 \mathrm{H}), 3.93(\mathrm{~d}, J=6.4 \mathrm{~Hz}, 1 \mathrm{H}), 4.1(\mathrm{t}, 2 \mathrm{H}), 5.25$ (s, 2H), $5.6(\mathrm{~d}, J=7.4 \mathrm{~Hz}, 1 \mathrm{H}), 7.05(\mathrm{t}, J=9.2 \mathrm{~Hz}, 2 \mathrm{H}), 7.25$ (d, J=7.4 Hz, 1H), 7.5 (t, $J=10.4 \mathrm{~Hz}, 1 \mathrm{H}), 7.58-7.64(\mathrm{~m}, 2 \mathrm{H}), 7.68$ (t, $J=9.8 \mathrm{~Hz}, 1 \mathrm{H}), 7.9(\mathrm{~d}, J=8.6 \mathrm{~Hz}, 1 \mathrm{H}), 8.1$ (s, $1 \mathrm{H}), 8.41(\mathrm{~d}, J=7.5 \mathrm{~Hz}, 1 \mathrm{H}), 8.65(\mathrm{~d}, J=9.2 \mathrm{~Hz}, 1 \mathrm{H}) .{ }^{\mathbf{1 3}} \mathbf{C}$ NMR $\left(\mathbf{1 0 0} \mathbf{M H z}, \mathbf{C D}_{\mathbf{3}} \mathbf{O D}, \boldsymbol{\delta}\right.$ ppm): 30.81, 44.96, 45.82, 61.60, 62.52, 71.81, 74.09, 78.72, 78.76, 81.30, 89.91, 116.89 , 117.12, 117.72, 119.06, 124.42, 124.90, 125.60, 130.20, 131.0, 131.28, 132.43, 132.93, 133.57, 135.59, 136.2, 144.91, 153.51. FTIR (KBr, $\left.\mathbf{~ c m}^{-\mathbf{1}}\right) ; 1341\left(v_{-\mathrm{S}=\mathrm{O}}\right), 2848$ $\left(v_{-\mathrm{C}-\mathrm{H}}\right), 2927\left(v_{\text {Ar-C-H}}\right)$, and $3425\left(v_{-\mathrm{O}-\mathrm{H}}\right)$. HRMS: Chemical Formula is $\mathrm{C}_{27} \mathrm{H}_{30} \mathrm{FN}_{5} \mathrm{O}_{9} \mathrm{~S}_{2}$ $\left[\mathrm{M}+\mathrm{Na}^{+}\right]$, calculated mass is 674.1360 , found is 674.1361 . 

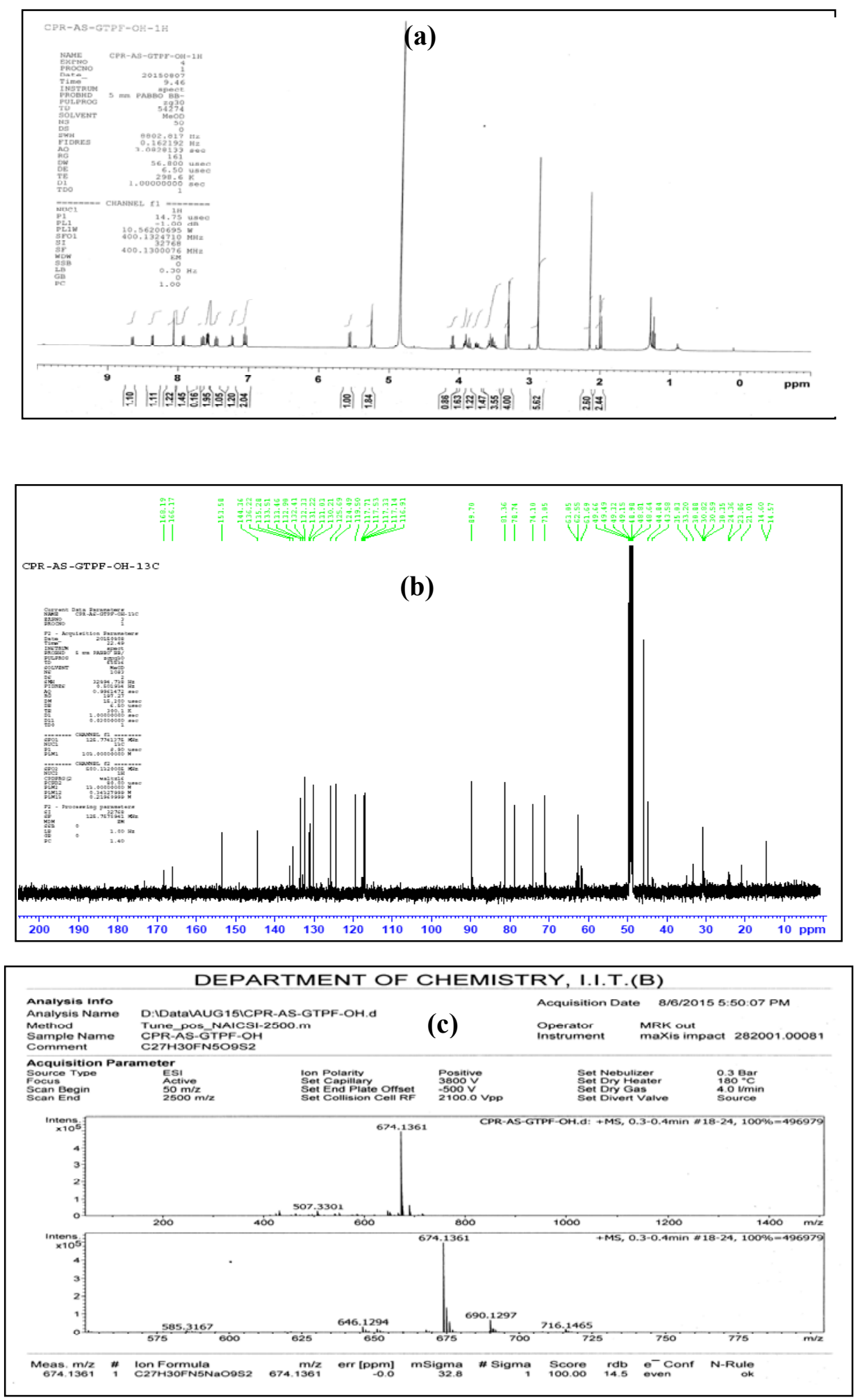

Figure S10: (a) ${ }^{1} \mathrm{H}$ NMR $\left(\mathrm{CD}_{3} \mathrm{OD}, 400 \mathrm{MHz}\right)(\mathrm{b}){ }^{13} \mathrm{C} \mathrm{NMR}\left(\mathrm{CD}_{3} \mathrm{OD}, 400 \mathrm{MHz}\right)(\mathrm{c}) \mathrm{HRMS}$ for ${ }^{1 \mathrm{~F}} \mathbf{L}_{\mathrm{OH}}$ 
S11. Synthesis and Characterization of ${ }^{0 \mathrm{~F}} \mathrm{~L}_{\mathrm{OH}}$ : The ${ }^{0 \mathrm{~F}} \mathrm{~L}_{\mathrm{OH}}$ has been synthesized by adopting the procedure that was used for ${ }^{5 \mathrm{~F}} \mathbf{L}_{\mathbf{O H}}$, but by using ${ }^{0 \mathrm{~F}} \mathbf{L}_{\mathbf{O A c}}(860 \mathrm{mg}, 0.01 \mathrm{mmol})$ in place of ${ }^{5 F} \mathbf{L}_{\mathbf{O A c}}$ in order to obtain the product in $64 \%$ yield as yellow solid. ${ }^{1} \mathbf{H}$ NMR (400 MHz, CD $\mathbf{C D D}_{3}$, $\boldsymbol{\delta}$ ppm): 2.85 (s, 6H), 3.3 (s, 2H), 3.49-3.55 (m, 2H), 3.72 (dd, $J=4.2,3.8$ $\mathrm{Hz}, 1 \mathrm{H}), 3.8(\mathrm{t}, J=8.4 \mathrm{~Hz}, 1 \mathrm{H}), 3.83(\mathrm{~d}, J=6.2 \mathrm{~Hz}, 1 \mathrm{H}), 4.01(\mathrm{t}, 2 \mathrm{H}), 5.36(\mathrm{~s}, 2 \mathrm{H}), 5.5$ (d, $J=$ $6.4 \mathrm{~Hz}, 1 \mathrm{H}), 7.26(\mathrm{~d}, \mathrm{~J}=7.2 \mathrm{~Hz}, 1 \mathrm{H}), 7.5$ (t, $J=11.4 \mathrm{~Hz}, 1 \mathrm{H}), 7.58-7.64(\mathrm{~m}, 2 \mathrm{H}), 7.68$ (t, $J=10.8 \mathrm{~Hz}, 1 \mathrm{H}), 8.05(\mathrm{~d}, J=8.4 \mathrm{~Hz}, 1 \mathrm{H}), 8.15(\mathrm{~s}, 1 \mathrm{H}), 8.35$ (d, $J=7.6 \mathrm{~Hz}, 1 \mathrm{H}), 8.63(\mathrm{~d}, J=8.2$ $\mathrm{Hz}, 1 \mathrm{H}) .{ }^{13} \mathbf{C}$ NMR (100 MHz, CD $\mathbf{3}$ OD, $\boldsymbol{\delta}$ ppm):43.91, 45.4, 61.4, 67.6, 70.3, 72.6, 75.3, 85.7, 115.5, 117.8, 123.2, 123.3, 123.7, 129.2, 129.7, 129.8, 132.4, 132.7, 133.5, 144.1, 144.0, 150.4, 152.3. FTIR (KBr, $\left.\mathbf{c m}^{-\mathbf{1}}\right)$; $1338\left(v_{-\mathrm{S}=\mathrm{O}}\right), 2852\left(v_{-\mathrm{C}-\mathrm{H}}\right), 2935\left(v_{\text {Ar-C-H}}\right)$, and 3435 ( $\left.v_{-\mathrm{O}-\mathrm{H}}\right)$. LRMS: Chemical Formula is $\mathrm{C}_{27} \mathrm{H}_{31} \mathrm{~N}_{5} \mathrm{O}_{9} \mathrm{~S}_{2}\left[\mathrm{M}+\mathrm{Na}^{+}\right]$, observed mass is 655.28.

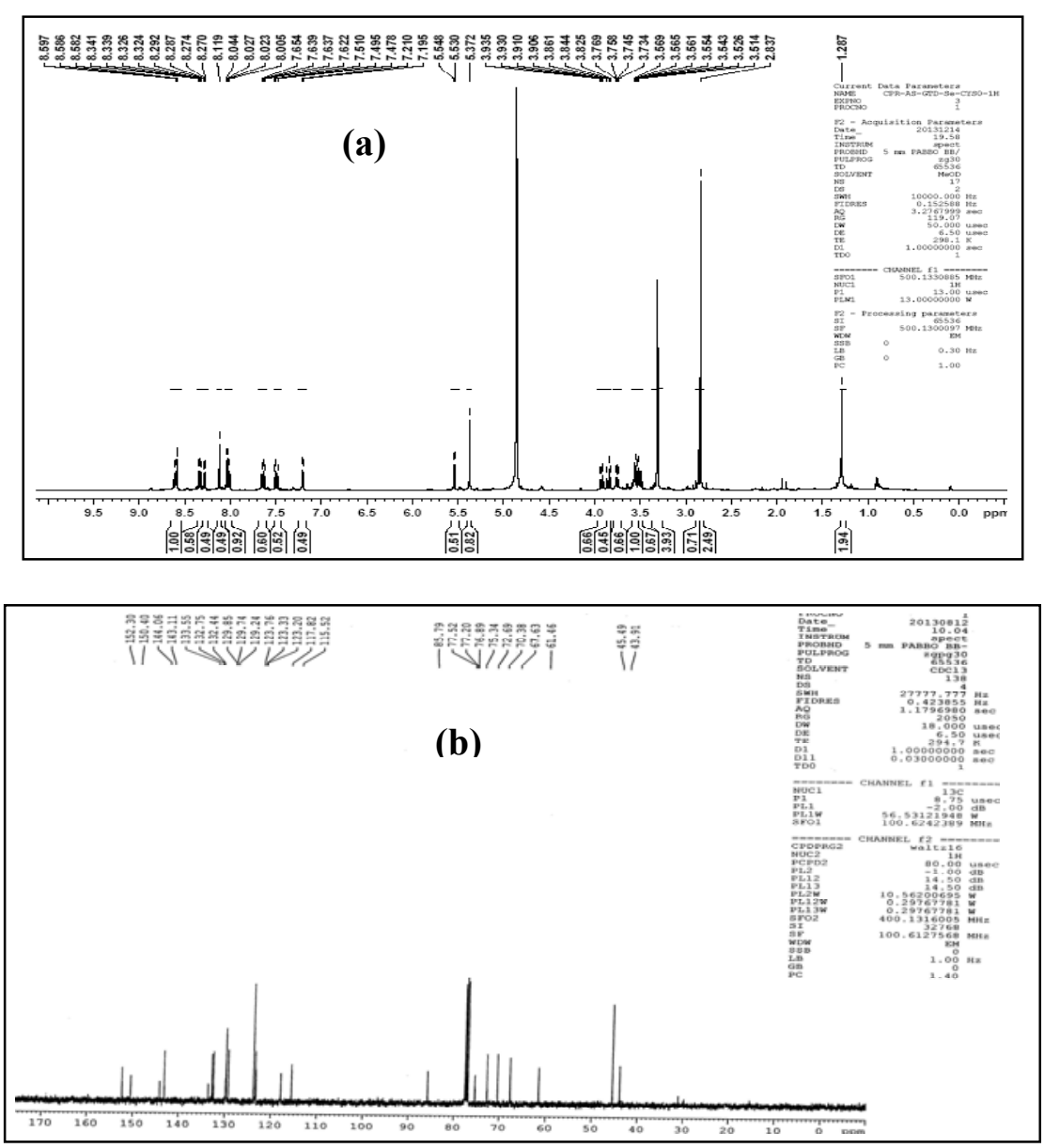




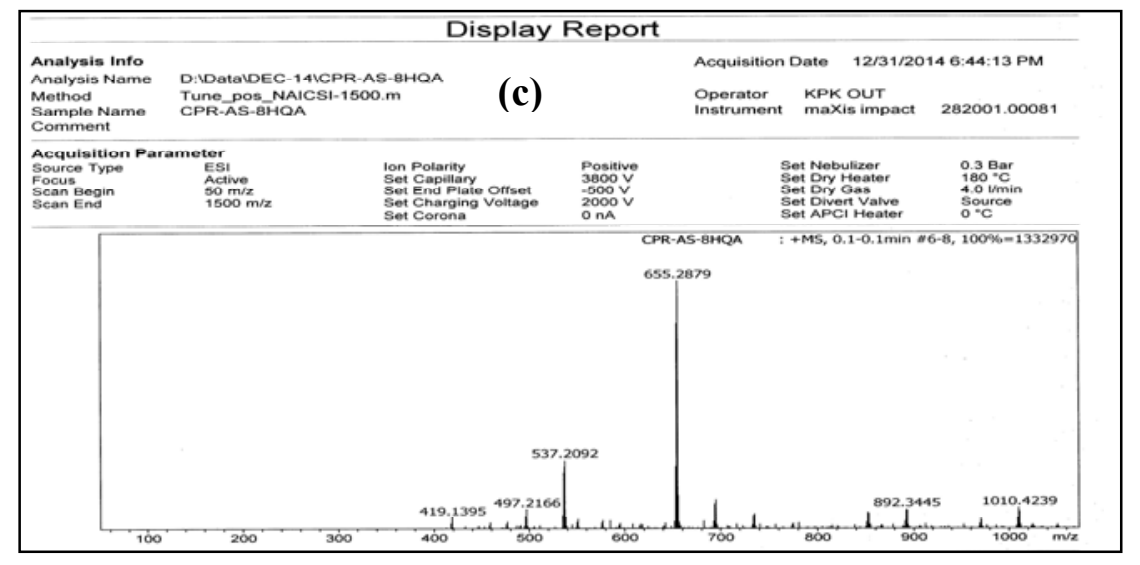

Figure S11: (a) ${ }^{1} \mathrm{H}$ NMR $\left(\mathrm{CD}_{3} \mathrm{OD}, 400 \mathrm{MHz}\right)\left(\right.$ b) ${ }^{13} \mathrm{C} \mathrm{NMR}\left(\mathrm{CDCl}_{3}, 400 \mathrm{MHz}\right)$ (c) LRMS for ${ }^{0 \mathrm{~F}} \mathbf{L}_{\mathbf{O H}}$.

S12. characterization of $\mathbf{L}_{1}$ : ${ }^{1} \mathbf{H}$ NMR (400 MHz, $\mathbf{D}_{\mathbf{2}} \mathbf{O}, \boldsymbol{\delta}$ ppm): 2.84 (s, 6H), 3.48-3.6 (m 2H), $3.7(\mathrm{dd}, J=3.4,2.8 \mathrm{~Hz}, 1 \mathrm{H}), 3.84$ (t, $J=13.8 \mathrm{~Hz}, 1 \mathrm{H}), 3.94(\mathrm{~d}, J=6.4 \mathrm{~Hz}, 1 \mathrm{H}), 4.2(\mathrm{~s}$, 2H) $5.37(\mathrm{~s}, 2 \mathrm{H}), 5.4(\mathrm{~d}, J=7.8 \mathrm{~Hz}, 1 \mathrm{H}), 7.25(\mathrm{~d}, J=7.8 \mathrm{~Hz}, 1 \mathrm{H}), 7.85(\mathrm{~s}, 1 \mathrm{H}), 8.2(\mathrm{~d}, J=$ $8.6 \mathrm{~Hz}, 1 \mathrm{H}), 8.31(\mathrm{~d}, J=8.2 \mathrm{~Hz}, 1 \mathrm{H}), 8.53(\mathrm{~d}, J=7.6 \mathrm{~Hz}, 1 \mathrm{H}) ;{ }^{13} \mathbf{C}$ NMR (100 MHz, $\left.\mathbf{C D C l}_{3}, \boldsymbol{\delta} \mathbf{p p m}\right): \quad 36.9,44.8,60.3,61.65,68.7,72.29,75.71,78.66,87.20,115.69,118.72$, $122.87,123.73,128.56,129.8,130.18,133.56,143.17,150.5$. ESI MS: Chemical Formula: $\mathrm{C}_{21} \mathrm{H}_{27} \mathrm{~N}_{5} \mathrm{O}_{7} \mathrm{~S}\left[\mathrm{M}+\mathrm{H}^{+}\right]$, calculated mass: 494.17, observed mass: 494.17 .

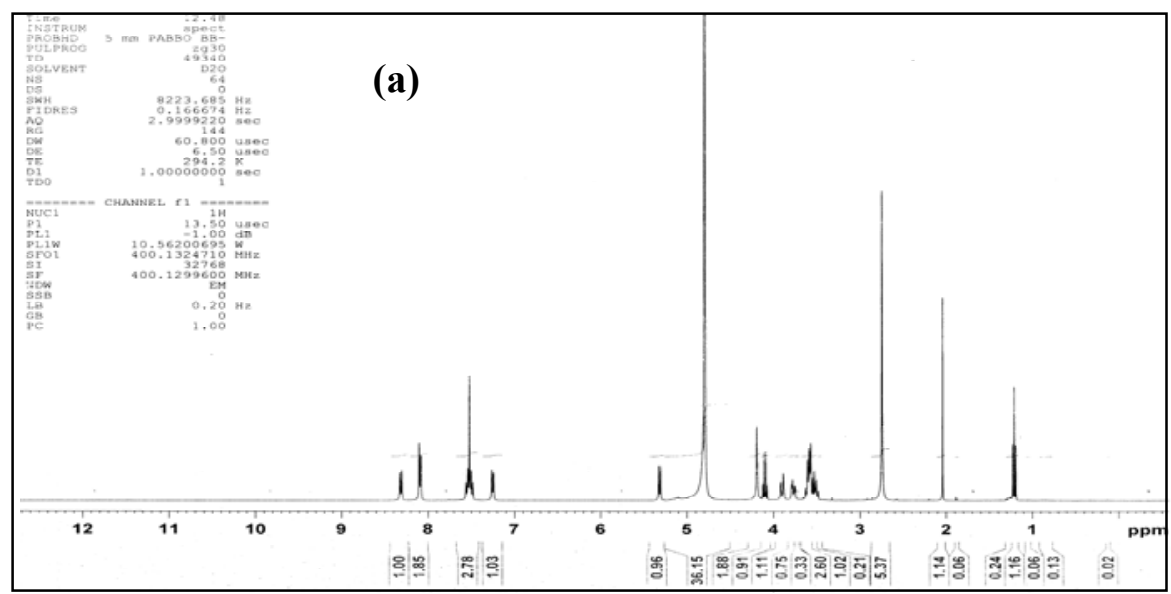



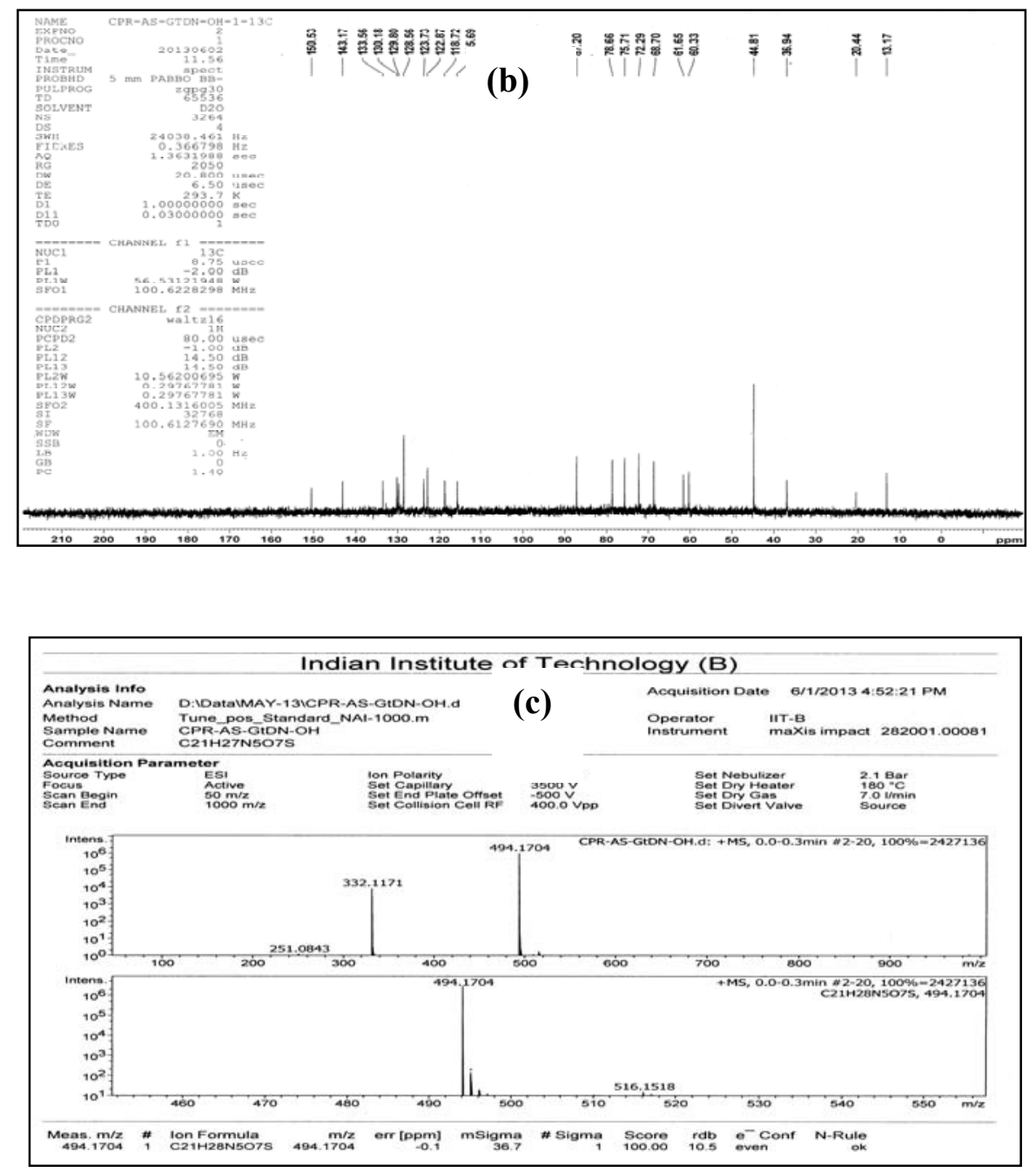

Figure S12: (a) ${ }^{1} \mathrm{H}$ NMR $\left(\mathrm{CD}_{3} \mathrm{OD}, 400 \mathrm{MHz}\right)(\mathrm{b}){ }^{13} \mathrm{C}$ NMR $\left(\mathrm{D}_{2} \mathrm{O}, 400 \mathrm{MHz}\right)(\mathrm{c}) \mathrm{HRMS}$ for $\mathbf{L}_{1}$. 
S13. Crystallographic data for ${ }^{5 \mathrm{~F}} \mathrm{~L}_{\mathrm{OAc}}$

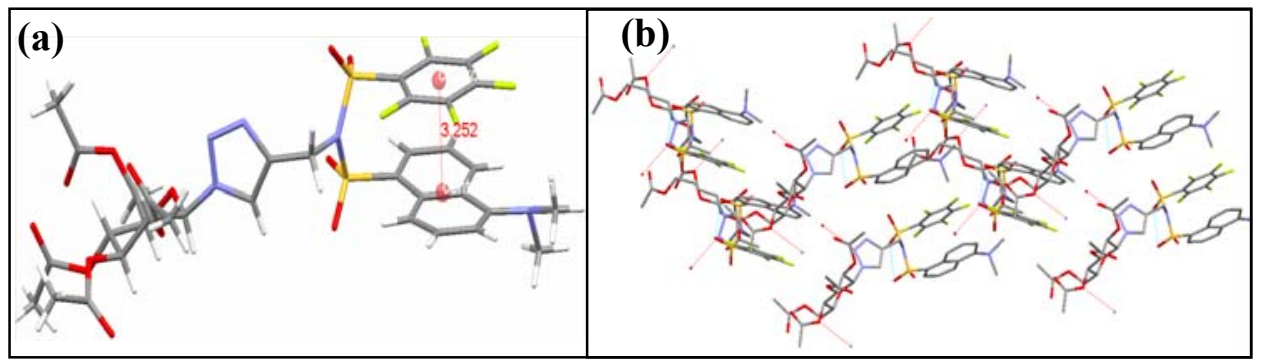

Figure S13. Crystal structure of ${ }^{5 \mathrm{~F}} \mathbf{L}_{\mathbf{O A c}}$ : (a) The diagram showing intramolecular $\pi---\pi$ interactions and the distance between them given in $\AA$. (b) Lattice diagram.

\section{Bond distances (Å)}

$\begin{array}{llll}\mathrm{S} 1-\mathrm{O} 13 & 1.423(3) & \mathrm{C} 23-\mathrm{H} 23 & 1.0000 \\ \mathrm{~S} 1-\mathrm{O} 39 & 1.426(3) & \mathrm{C} 25-\mathrm{H} 25 & 0.9500 \\ \mathrm{~S} 1-\mathrm{N} 10 & 1.706(3) & \mathrm{C} 25-\mathrm{C} 28 & 1.367(6) \\ \mathrm{S} 1-\mathrm{C} 17 & 1.761(4) & \mathrm{C} 25-\mathrm{C} 40 & 1.401(6) \\ \mathrm{S} 2-\mathrm{O} 15 & 1.416(3) & \mathrm{C} 26-\mathrm{H} 26 & 0.9500 \\ \mathrm{~S} 2-\mathrm{O} 24 & 1.431(3) & \mathrm{C} 26-\mathrm{C} 37 & 1.415(6) \\ \mathrm{S} 2-\mathrm{N} 10 & 1.649(3) & \mathrm{C} 26-\mathrm{C} 43 & 1.369(6) \\ \mathrm{S} 2-\mathrm{C} 14 & 1.777(4) & \mathrm{C} 27-\mathrm{C} 38 & 1.436(6) \\ \mathrm{F} 3-\mathrm{C} 46 & 1.335(5) & \mathrm{C} 28-\mathrm{H} 28 & 0.9500 \\ \mathrm{~F} 4-\mathrm{C} 19 & 1.342(4) & \mathrm{C} 28-\mathrm{C} 38 & 1.431(6) \\ \mathrm{F} 5-\mathrm{C} 11 & 1.337(5) & \mathrm{C} 30-\mathrm{C} 46 & 1.375(6) \\ \mathrm{F} 6-\mathrm{C} 30 & 1.343(4) & \mathrm{C} 31-\mathrm{H} 31 & 1.0000 \\ \mathrm{~F} 7-\mathrm{C} 52 & 1.339(4) & \mathrm{C} 31-\mathrm{C} 53 & 1.517(5) \\ \mathrm{O} 9-\mathrm{C} 20 & 1.431(5) & \mathrm{C} 35-\mathrm{H} 35 \mathrm{~A} & 0.9900 \\ \mathrm{O} 9-\mathrm{C} 41 & 1.378(5) & \mathrm{C} 35-\mathrm{H} 35 \mathrm{~B} & 0.9900 \\ \mathrm{O} 12-\mathrm{C} 31 & 1.434(4) & \mathrm{C} 35-\mathrm{C} 50 & 1.502(5) \\ \mathrm{O} 12-\mathrm{C} 47 & 1.354(5) & \mathrm{C} 37-\mathrm{C} 38 & 1.429(5) \\ \mathrm{O} 16-\mathrm{C} 53 & 1.430(5) & \mathrm{C} 40-\mathrm{H} 40 & 0.9500 \\ \mathrm{O} 16-\mathrm{C} 54 & 1.351(5) & \mathrm{C} 41-\mathrm{C} 59 & 1.479(6) \\ & & & \end{array}$




\begin{tabular}{|c|c|c|c|}
\hline $\mathrm{O} 18-\mathrm{C} 42$ & $1.440(5)$ & $\mathrm{C} 42-\mathrm{H} 42 \mathrm{~A}$ & 0.9900 \\
\hline $\mathrm{O} 18-\mathrm{C} 58$ & $1.344(5)$ & $\mathrm{C} 42-\mathrm{H} 42 \mathrm{~B}$ & 0.9900 \\
\hline $\mathrm{O} 21-\mathrm{C} 41$ & $1.194(5)$ & $\mathrm{C} 42-\mathrm{C} 51$ & $1.512(5)$ \\
\hline $\mathrm{O} 29-\mathrm{C} 54$ & $1.195(5)$ & $\mathrm{C} 43-\mathrm{H} 43$ & 0.9500 \\
\hline $\mathrm{O} 33-\mathrm{C} 47$ & $1.204(5)$ & $\mathrm{C} 44-\mathrm{H} 44 \mathrm{~A}$ & 0.9800 \\
\hline $\mathrm{O} 34-\mathrm{C} 23$ & $1.407(5)$ & $\mathrm{C} 44-\mathrm{H} 44 \mathrm{~B}$ & 0.9800 \\
\hline $\mathrm{O} 34-\mathrm{C} 51$ & $1.437(4)$ & $\mathrm{C} 44-\mathrm{H} 44 \mathrm{C}$ & 0.9800 \\
\hline $\mathrm{O} 55-\mathrm{C} 58$ & $1.207(6)$ & $\mathrm{C} 45-\mathrm{H} 45 \mathrm{~A}$ & 0.9800 \\
\hline $\mathrm{N} 8-\mathrm{C} 27$ & $1.427(5)$ & $\mathrm{C} 45-\mathrm{H} 45 \mathrm{~B}$ & 0.9800 \\
\hline $\mathrm{N} 8-\mathrm{C} 44$ & $1.467(6)$ & $\mathrm{C} 45-\mathrm{H} 45 \mathrm{C}$ & 0.9800 \\
\hline $\mathrm{N} 8-\mathrm{C} 45$ & $1.459(5)$ & $\mathrm{C} 47-\mathrm{C} 57$ & $1.498(6)$ \\
\hline $\mathrm{N} 10-\mathrm{C} 35$ & $1.503(5)$ & $\mathrm{C} 50-\mathrm{C} 61$ & $1.360(5)$ \\
\hline $\mathrm{N} 32-\mathrm{N} 36$ & $1.314(4)$ & C51-H51 & 1.0000 \\
\hline N32-N49 & $1.349(5)$ & $\mathrm{C} 51-\mathrm{C} 53$ & $1.545(5)$ \\
\hline N36-C50 & $1.367(5)$ & C53-H53 & 1.0000 \\
\hline $\mathrm{N} 49-\mathrm{C} 23$ & $1.438(5)$ & $\mathrm{C} 54-\mathrm{C} 56$ & $1.498(6)$ \\
\hline N49-C61 & $1.342(5)$ & С56-H56A & 0.9800 \\
\hline $\mathrm{C} 11-\mathrm{C} 19$ & $1.384(6)$ & $\mathrm{C} 56-\mathrm{H} 56 \mathrm{~B}$ & 0.9800 \\
\hline $\mathrm{C} 11-\mathrm{C} 52$ & $1.375(6)$ & $\mathrm{C} 56-\mathrm{H} 56 \mathrm{C}$ & 0.9800 \\
\hline $\mathrm{C} 14-\mathrm{C} 46$ & $1.394(5)$ & C57-H57A & 0.9800 \\
\hline $\mathrm{C} 14-\mathrm{C} 52$ & $1.385(6)$ & С $57-\mathrm{H} 57 \mathrm{~B}$ & 0.9800 \\
\hline $\mathrm{C} 17-\mathrm{C} 37$ & $1.424(6)$ & $\mathrm{C} 57-\mathrm{H} 57 \mathrm{C}$ & 0.9800 \\
\hline $\mathrm{C} 17-\mathrm{C} 40$ & $1.374(6)$ & $\mathrm{C} 58-\mathrm{C} 60$ & $1.503(7)$ \\
\hline C19-C30 & $1.371(6)$ & С59-H59A & 0.9800 \\
\hline $\mathrm{C} 20-\mathrm{H} 20$ & 1.0000 & С59-Н59B & 0.9800 \\
\hline $\mathrm{C} 20-\mathrm{C} 23$ & $1.526(5)$ & С59-H59C & 0.9800 \\
\hline $\mathrm{C} 20-\mathrm{C} 31$ & $1.526(5)$ & $\mathrm{C} 60-\mathrm{H} 60 \mathrm{~A}$ & 0.9800 \\
\hline $\mathrm{C} 22-\mathrm{H} 22$ & 0.9500 & $\mathrm{C} 60-\mathrm{H} 60 \mathrm{~B}$ & 0.9800 \\
\hline $\mathrm{C} 22-\mathrm{C} 27$ & $1.369(6)$ & $\mathrm{C} 60-\mathrm{H} 60 \mathrm{C}$ & 0.9800 \\
\hline
\end{tabular}




\section{Bond angles ( $\AA$ )}

$\begin{array}{llll}\mathrm{O} 13-\mathrm{S} 1-\mathrm{O} 39 & 118.6(2) & \mathrm{C} 37-\mathrm{C} 38-\mathrm{C} 27 & 119.4(4) \\ \mathrm{O} 13-\mathrm{S} 1-\mathrm{N} 10 & 109.73(19) & \mathrm{C} 37-\mathrm{C} 38-\mathrm{C} 28 & 119.6(4) \\ \mathrm{O} 13-\mathrm{S} 1-\mathrm{C} 17 & 108.7(2) & \mathrm{C} 17-\mathrm{C} 40-\mathrm{C} 25 & 120.1(4) \\ \mathrm{O} 39-\mathrm{S} 1-\mathrm{N} 10 & 102.86(18) & \mathrm{C} 17-\mathrm{C} 40-\mathrm{H} 40 & 120.0 \\ \mathrm{O} 39-\mathrm{S} 1-\mathrm{C} 17 & 111.22(18) & \mathrm{C} 25-\mathrm{C} 40-\mathrm{H} 40 & 120.0 \\ \mathrm{~N} 10-\mathrm{S} 1-\mathrm{C} 17 & 104.77(18) & \mathrm{O} 9-\mathrm{C} 41-\mathrm{C} 59 & 110.9(4) \\ \mathrm{O} 15-\mathrm{S} 2-\mathrm{O} 24 & 120.60(19) & \mathrm{O} 21-\mathrm{C} 41-\mathrm{O} 9 & 123.3(4) \\ \mathrm{O} 15-\mathrm{S} 2-\mathrm{N} 10 & 109.41(18) & \mathrm{O} 21-\mathrm{C} 41-\mathrm{C} 59 & 125.8(4) \\ \mathrm{O} 15-\mathrm{S} 2-\mathrm{C} 14 & 108.02(18) & \mathrm{O} 18-\mathrm{C} 42-\mathrm{H} 42 \mathrm{~A} & 109.6 \\ \mathrm{O} 24-\mathrm{S} 2-\mathrm{N} 10 & 106.05(17) & \mathrm{O} 18-\mathrm{C} 42-\mathrm{H} 42 \mathrm{~B} & 109.6 \\ \mathrm{O} 24-\mathrm{S} 2-\mathrm{C} 14 & 107.75(18) & \mathrm{O} 18-\mathrm{C} 42-\mathrm{C} 51 & 110.2(4) \\ \mathrm{N} 10-\mathrm{S} 2-\mathrm{C} 14 & 103.76(17) & \mathrm{H} 42 \mathrm{~A}-\mathrm{C} 42-\mathrm{H} 42 \mathrm{~B} & 108.1 \\ \mathrm{C} 41-\mathrm{O} 9-\mathrm{C} 20 & 116.5(3) & \mathrm{C} 51-\mathrm{C} 42-\mathrm{H} 42 \mathrm{~A} & 109.6 \\ \mathrm{C} 47-\mathrm{O} 12-\mathrm{C} 31 & 116.7(3) & \mathrm{C} 51-\mathrm{C} 42-\mathrm{H} 42 \mathrm{~B} & 109.6 \\ \mathrm{C} 54-\mathrm{O} 16-\mathrm{C} 53 & 119.1(3) & \mathrm{C} 22-\mathrm{C} 43-\mathrm{H} 43 & 119.2 \\ \mathrm{C} 58-\mathrm{O} 18-\mathrm{C} 42 & 117.3(4) & \mathrm{C} 26-\mathrm{C} 43-\mathrm{C} 22 & 121.7(4) \\ \mathrm{C} 23-\mathrm{O} 34-\mathrm{C} 51 & 111.8(3) & \mathrm{C} 26-\mathrm{C} 43-\mathrm{H} 43 & 119.2 \\ \mathrm{C} 27-\mathrm{N} 8-\mathrm{C} 44 & 112.9(3) & \mathrm{N} 8-\mathrm{C} 44-\mathrm{H} 44 \mathrm{~A} & 109.5 \\ \mathrm{C} 27-\mathrm{N} 8-\mathrm{C} 45 & 115.0(4) & \mathrm{N} 8-\mathrm{C} 44-\mathrm{H} 44 \mathrm{~B} & 109.5 \\ \mathrm{C} 45-\mathrm{N} 8-\mathrm{C} 44 & 111.0(4) & \mathrm{N} 8-\mathrm{C} 44-\mathrm{H} 44 \mathrm{C} & 109.5 \\ \mathrm{~S} 2-\mathrm{N} 10-\mathrm{S} 1 & 120.3(2) & \mathrm{H} 44 \mathrm{~A}-\mathrm{C} 44-\mathrm{H} 44 \mathrm{~B} & 109.5 \\ \mathrm{C} 35-\mathrm{N} 10-\mathrm{S} 1 & 117.8(2) & \mathrm{H} 44 \mathrm{~A}-\mathrm{C} 44-\mathrm{H} 44 \mathrm{C} & 109.5 \\ \mathrm{C} 35-\mathrm{N} 10-\mathrm{S} 2 & 121.1(3) & \mathrm{H} 44 \mathrm{~B}-\mathrm{C} 44-\mathrm{H} 44 \mathrm{C} & 109.5 \\ \mathrm{~N} 36-\mathrm{N} 32-\mathrm{N} 49 & 106.8(3) & \mathrm{N} 8-\mathrm{C} 45-\mathrm{H} 45 \mathrm{~A} & 109.5 \\ \mathrm{~N} 32-\mathrm{N} 36-\mathrm{C} 50 & 108.6(3) & \mathrm{N} 8-\mathrm{C} 45-\mathrm{H} 45 \mathrm{~B} & 109.5 \\ \mathrm{~N} 32-\mathrm{N} 49-\mathrm{C} 23 & 121.2(3) & \mathrm{N} 8-\mathrm{C} 45-\mathrm{H} 45 \mathrm{C} & 109.5 \\ \mathrm{C} 49-\mathrm{N} 32 & 111.2(3) & \mathrm{H} 45 \mathrm{~A}-\mathrm{C} 45-\mathrm{H} 45 \mathrm{~B} & 109.5 \\ \mathrm{C} 233 & 127.6(3) & \mathrm{H} 45 \mathrm{~A}-\mathrm{C} 45-\mathrm{H} 45 \mathrm{C} & 109.5\end{array}$




\begin{tabular}{|c|c|c|c|}
\hline $\mathrm{F} 5-\mathrm{C} 11-\mathrm{C} 19$ & $120.4(4)$ & $\mathrm{H} 45 \mathrm{~B}-\mathrm{C} 45-\mathrm{H} 45 \mathrm{C}$ & 109.5 \\
\hline $\mathrm{F} 5-\mathrm{C} 11-\mathrm{C} 52$ & $120.7(4)$ & $\mathrm{F} 3-\mathrm{C} 46-\mathrm{C} 14$ & $121.7(4)$ \\
\hline $\mathrm{C} 52-\mathrm{C} 11-\mathrm{C} 19$ & $118.9(4)$ & $\mathrm{F} 3-\mathrm{C} 46-\mathrm{C} 30$ & $117.3(4)$ \\
\hline $\mathrm{C} 46-\mathrm{C} 14-\mathrm{S} 2$ & $123.0(3)$ & $\mathrm{C} 30-\mathrm{C} 46-\mathrm{C} 14$ & $120.9(4)$ \\
\hline $\mathrm{C} 52-\mathrm{C} 14-\mathrm{S} 2$ & $119.6(3)$ & $\mathrm{O} 12-\mathrm{C} 47-\mathrm{C} 57$ & $111.1(4)$ \\
\hline $\mathrm{C} 52-\mathrm{C} 14-\mathrm{C} 46$ & $117.4(4)$ & $\mathrm{O} 33-\mathrm{C} 47-\mathrm{O} 12$ & $124.1(4)$ \\
\hline $\mathrm{C} 37-\mathrm{C} 17-\mathrm{S} 1$ & $121.6(3)$ & $\mathrm{O} 33-\mathrm{C} 47-\mathrm{C} 57$ & $124.8(4)$ \\
\hline $\mathrm{C} 40-\mathrm{C} 17-\mathrm{S} 1$ & $115.5(3)$ & $\mathrm{N} 36-\mathrm{C} 50-\mathrm{C} 35$ & $122.1(3)$ \\
\hline $\mathrm{C} 40-\mathrm{C} 17-\mathrm{C} 37$ & $122.9(4)$ & $\mathrm{C} 61-\mathrm{C} 50-\mathrm{N} 36$ & $108.6(3)$ \\
\hline $\mathrm{F} 4-\mathrm{C} 19-\mathrm{C} 11$ & $120.0(4)$ & $\mathrm{C} 61-\mathrm{C} 50-\mathrm{C} 35$ & $129.2(4)$ \\
\hline $\mathrm{F} 4-\mathrm{C} 19-\mathrm{C} 30$ & $119.7(4)$ & $\mathrm{O} 34-\mathrm{C} 51-\mathrm{C} 42$ & $107.5(3)$ \\
\hline $\mathrm{C} 30-\mathrm{C} 19-\mathrm{C} 11$ & $120.3(4)$ & $\mathrm{O} 34-\mathrm{C} 51-\mathrm{H} 51$ & 108.4 \\
\hline $\mathrm{O} 9-\mathrm{C} 20-\mathrm{H} 20$ & 109.7 & $\mathrm{O} 34-\mathrm{C} 51-\mathrm{C} 53$ & $110.4(3)$ \\
\hline $\mathrm{O} 9-\mathrm{C} 20-\mathrm{C} 23$ & $109.3(3)$ & $\mathrm{C} 42-\mathrm{C} 51-\mathrm{H} 51$ & 108.4 \\
\hline $\mathrm{O} 9-\mathrm{C} 20-\mathrm{C} 31$ & $111.7(3)$ & $\mathrm{C} 42-\mathrm{C} 51-\mathrm{C} 53$ & $113.7(3)$ \\
\hline $\mathrm{C} 23-\mathrm{C} 20-\mathrm{H} 20$ & 109.7 & $\mathrm{C} 53-\mathrm{C} 51-\mathrm{H} 51$ & 108.4 \\
\hline $\mathrm{C} 23-\mathrm{C} 20-\mathrm{C} 31$ & $106.6(3)$ & $\mathrm{F} 7-\mathrm{C} 52-\mathrm{C} 11$ & $117.8(4)$ \\
\hline $\mathrm{C} 31-\mathrm{C} 20-\mathrm{H} 20$ & 109.7 & $\mathrm{~F} 7-\mathrm{C} 52-\mathrm{C} 14$ & $120.0(4)$ \\
\hline $\mathrm{C} 27-\mathrm{C} 22-\mathrm{H} 22$ & 119.7 & $\mathrm{C} 11-\mathrm{C} 52-\mathrm{C} 14$ & $122.2(4)$ \\
\hline $\mathrm{C} 27-\mathrm{C} 22-\mathrm{C} 43$ & $120.6(4)$ & $\mathrm{O} 16-\mathrm{C} 53-\mathrm{C} 31$ & $110.2(3)$ \\
\hline $\mathrm{C} 43-\mathrm{C} 22-\mathrm{H} 22$ & 119.7 & $\mathrm{O} 16-\mathrm{C} 53-\mathrm{C} 51$ & $105.4(3)$ \\
\hline $\mathrm{O} 34-\mathrm{C} 23-\mathrm{N} 49$ & $109.5(3)$ & $\mathrm{O} 16-\mathrm{C} 53-\mathrm{H} 53$ & 110.3 \\
\hline $\mathrm{O} 34-\mathrm{C} 23-\mathrm{C} 20$ & $107.9(3)$ & $\mathrm{C} 31-\mathrm{C} 53-\mathrm{C} 51$ & $110.3(3)$ \\
\hline $\mathrm{O} 34-\mathrm{C} 23-\mathrm{H} 23$ & 108.8 & $\mathrm{C} 31-\mathrm{C} 53-\mathrm{H} 53$ & 110.3 \\
\hline $\mathrm{N} 49-\mathrm{C} 23-\mathrm{C} 20$ & $113.0(3)$ & $\mathrm{C} 51-\mathrm{C} 53-\mathrm{H} 53$ & 110.3 \\
\hline $\mathrm{N} 49-\mathrm{C} 23-\mathrm{H} 23$ & 108.8 & $\mathrm{O} 16-\mathrm{C} 54-\mathrm{C} 56$ & $109.8(4)$ \\
\hline $\mathrm{C} 20-\mathrm{C} 23-\mathrm{H} 23$ & 108.8 & $\mathrm{O} 29-\mathrm{C} 54-\mathrm{O} 16$ & $124.6(4)$ \\
\hline $\mathrm{C} 28-\mathrm{C} 25-\mathrm{H} 25$ & 120.2 & $\mathrm{O} 29-\mathrm{C} 54-\mathrm{C} 56$ & $125.6(4)$ \\
\hline $\mathrm{C} 28-\mathrm{C} 25-\mathrm{C} 40$ & $119.7(4)$ & $\mathrm{C} 54-\mathrm{C} 56-\mathrm{H} 56 \mathrm{~A}$ & 109.5 \\
\hline
\end{tabular}




\begin{tabular}{|c|c|c|c|}
\hline $\mathrm{C} 40-\mathrm{C} 25-\mathrm{H} 25$ & 120.2 & $\mathrm{C} 54-\mathrm{C} 56-\mathrm{H} 56 \mathrm{~B}$ & 109.5 \\
\hline $\mathrm{C} 37-\mathrm{C} 26-\mathrm{H} 26$ & 120.2 & $\mathrm{C} 54-\mathrm{C} 56-\mathrm{H} 56 \mathrm{C}$ & 109.5 \\
\hline $\mathrm{C} 43-\mathrm{C} 26-\mathrm{H} 26$ & 120.2 & $\mathrm{H} 56 \mathrm{~A}-\mathrm{C} 56-\mathrm{H} 56 \mathrm{~B}$ & 109.5 \\
\hline $\mathrm{C} 43-\mathrm{C} 26-\mathrm{C} 37$ & $119.7(4)$ & $\mathrm{H} 56 \mathrm{~A}-\mathrm{C} 56-\mathrm{H} 56 \mathrm{C}$ & 109.5 \\
\hline $\mathrm{N} 8-\mathrm{C} 27-\mathrm{C} 38$ & $117.3(4)$ & $\mathrm{H} 56 \mathrm{~B}-\mathrm{C} 56-\mathrm{H} 56 \mathrm{C}$ & 109.5 \\
\hline $\mathrm{C} 22-\mathrm{C} 27-\mathrm{N} 8$ & $123.3(4)$ & C47-C57-H57A & 109.5 \\
\hline $\mathrm{C} 22-\mathrm{C} 27-\mathrm{C} 38$ & $119.3(4)$ & C47-C57-H57B & 109.5 \\
\hline $\mathrm{C} 25-\mathrm{C} 28-\mathrm{H} 28$ & 119.3 & $\mathrm{C} 47-\mathrm{C} 57-\mathrm{H} 57 \mathrm{C}$ & 109.5 \\
\hline $\mathrm{C} 25-\mathrm{C} 28-\mathrm{C} 38$ & $121.4(4)$ & $\mathrm{H} 57 \mathrm{~A}-\mathrm{C} 57-\mathrm{H} 57 \mathrm{~B}$ & 109.5 \\
\hline $\mathrm{C} 38-\mathrm{C} 28-\mathrm{H} 28$ & 119.3 & $\mathrm{H} 57 \mathrm{~A}-\mathrm{C} 57-\mathrm{H} 57 \mathrm{C}$ & 109.5 \\
\hline $\mathrm{F} 6-\mathrm{C} 30-\mathrm{C} 19$ & $119.5(4)$ & $\mathrm{H} 57 \mathrm{~B}-\mathrm{C} 57-\mathrm{H} 57 \mathrm{C}$ & 109.5 \\
\hline $\mathrm{F} 6-\mathrm{C} 30-\mathrm{C} 46$ & $120.2(4)$ & $\mathrm{O} 18-\mathrm{C} 58-\mathrm{C} 60$ & $110.4(4)$ \\
\hline $\mathrm{C} 19-\mathrm{C} 30-\mathrm{C} 46$ & $120.3(4)$ & $\mathrm{O} 55-\mathrm{C} 58-\mathrm{O} 18$ & $123.8(5)$ \\
\hline $\mathrm{O} 12-\mathrm{C} 31-\mathrm{C} 20$ & $109.5(3)$ & $\mathrm{O} 55-\mathrm{C} 58-\mathrm{C} 60$ & $125.8(5)$ \\
\hline $\mathrm{O} 12-\mathrm{C} 31-\mathrm{H} 31$ & 110.3 & $\mathrm{C} 41-\mathrm{C} 59-\mathrm{H} 59 \mathrm{~A}$ & 109.5 \\
\hline $\mathrm{O} 12-\mathrm{C} 31-\mathrm{C} 53$ & $109.1(3)$ & $\mathrm{C} 41-\mathrm{C} 59-\mathrm{H} 59 \mathrm{~B}$ & 109.5 \\
\hline $\mathrm{C} 20-\mathrm{C} 31-\mathrm{H} 31$ & 110.3 & $\mathrm{C} 41-\mathrm{C} 59-\mathrm{H} 59 \mathrm{C}$ & 109.5 \\
\hline $\mathrm{C} 53-\mathrm{C} 31-\mathrm{C} 20$ & $107.3(3)$ & $\mathrm{H} 59 \mathrm{~A}-\mathrm{C} 59-\mathrm{H} 59 \mathrm{~B}$ & 109.5 \\
\hline $\mathrm{C} 53-\mathrm{C} 31-\mathrm{H} 31$ & 110.3 & $\mathrm{H} 59 \mathrm{~A}-\mathrm{C} 59-\mathrm{H} 59 \mathrm{C}$ & 109.5 \\
\hline $\mathrm{N} 10-\mathrm{C} 35-\mathrm{H} 35 \mathrm{~A}$ & 109.0 & $\mathrm{H} 59 \mathrm{~B}-\mathrm{C} 59-\mathrm{H} 59 \mathrm{C}$ & 109.5 \\
\hline $\mathrm{N} 10-\mathrm{C} 35-\mathrm{H} 35 \mathrm{~B}$ & 109.0 & $\mathrm{C} 58-\mathrm{C} 60-\mathrm{H} 60 \mathrm{~A}$ & 109.5 \\
\hline $\mathrm{H} 35 \mathrm{~A}-\mathrm{C} 35-\mathrm{H} 35 \mathrm{~B}$ & 107.8 & $\mathrm{C} 58-\mathrm{C} 60-\mathrm{H} 60 \mathrm{~B}$ & 109.5 \\
\hline $\mathrm{C} 50-\mathrm{C} 35-\mathrm{N} 10$ & $113.1(3)$ & $\mathrm{C} 58-\mathrm{C} 60-\mathrm{H} 60 \mathrm{C}$ & 109.5 \\
\hline $\mathrm{C} 50-\mathrm{C} 35-\mathrm{H} 35 \mathrm{~A}$ & 109.0 & $\mathrm{H} 60 \mathrm{~A}-\mathrm{C} 60-\mathrm{H} 60 \mathrm{~B}$ & 109.5 \\
\hline $\mathrm{C} 50-\mathrm{C} 35-\mathrm{H} 35 \mathrm{~B}$ & 109.0 & $\mathrm{H} 60 \mathrm{~A}-\mathrm{C} 60-\mathrm{H} 60 \mathrm{C}$ & 109.5 \\
\hline $\mathrm{C} 17-\mathrm{C} 37-\mathrm{C} 38$ & $116.3(4)$ & $\mathrm{H} 60 \mathrm{~B}-\mathrm{C} 60-\mathrm{H} 60 \mathrm{C}$ & 109.5 \\
\hline $\mathrm{C} 26-\mathrm{C} 37-\mathrm{C} 17$ & $124.6(4)$ & $\mathrm{N} 49-\mathrm{C} 61-\mathrm{C} 50$ & $104.7(4)$ \\
\hline $\mathrm{C} 26-\mathrm{C} 37-\mathrm{C} 38$ & $119.1(4)$ & $\mathrm{N} 49-\mathrm{C} 61-\mathrm{H} 61$ & 127.6 \\
\hline $\mathrm{C} 28-\mathrm{C} 38-\mathrm{C} 27$ & $120.9(4)$ & $\mathrm{C} 50-\mathrm{C} 61-\mathrm{H} 61$ & 127.6 \\
\hline
\end{tabular}




\section{S14. pH Optimization for the reaction of ${ }^{5 \mathrm{~F}} \mathrm{~L}_{\mathrm{OH}}$ with $\mathrm{CN}^{-}$}
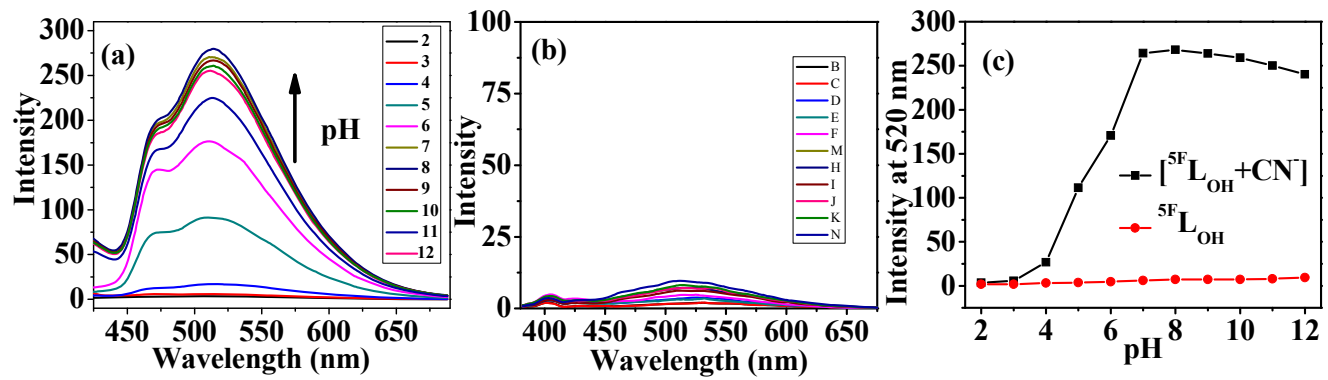

Figure S14. (a) The effect of $\mathrm{pH}$ on the fluorescence emission intensity upon reaction of (a) ${ }^{5 \mathrm{~F}} \mathbf{L}_{\mathrm{OH}}(5 \mu \mathrm{M})$ with $\mathrm{CN}^{-}(100 \mu \mathrm{M})\left(\right.$ b) ${ }^{5 \mathrm{~F}} \mathbf{L}_{\mathbf{O H}}(5 \mu \mathrm{M})$ when excited at $360 \mathrm{~nm}$ in aqueous acetonitrile buffer. (c) Plot showing the fluorescence intensity at $520 \mathrm{~nm} \mathrm{Vs} \mathrm{pH}$ for the reaction of $\left[{ }^{5} \mathbf{L}_{\mathbf{O H}}\right]$ with $\mathrm{CN}^{-}$.

\section{S15. Fluorescence studies of ${ }^{5 \mathrm{~F}} \mathrm{~L}_{\mathrm{OH}}$ with anions and metal ions}
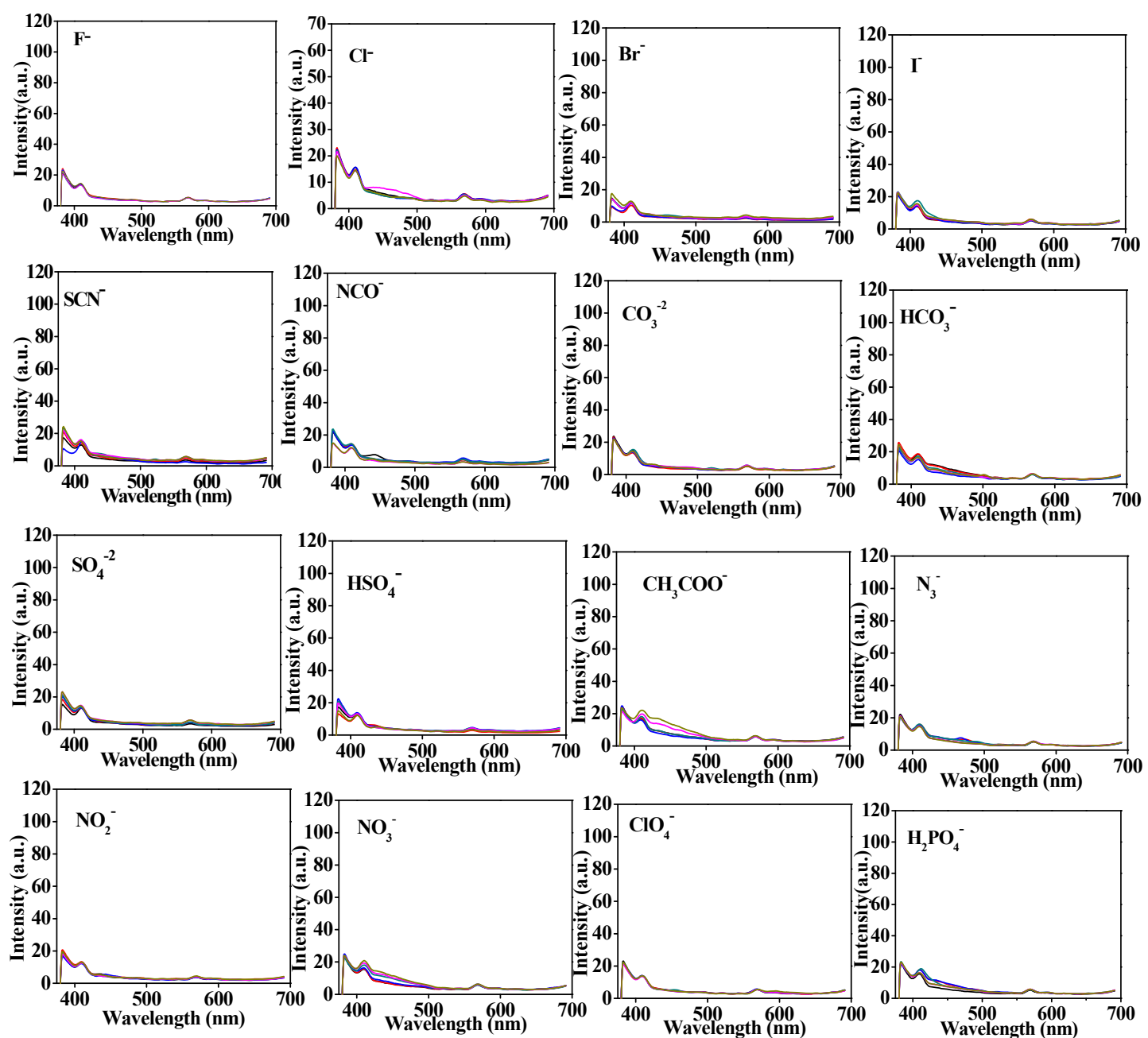

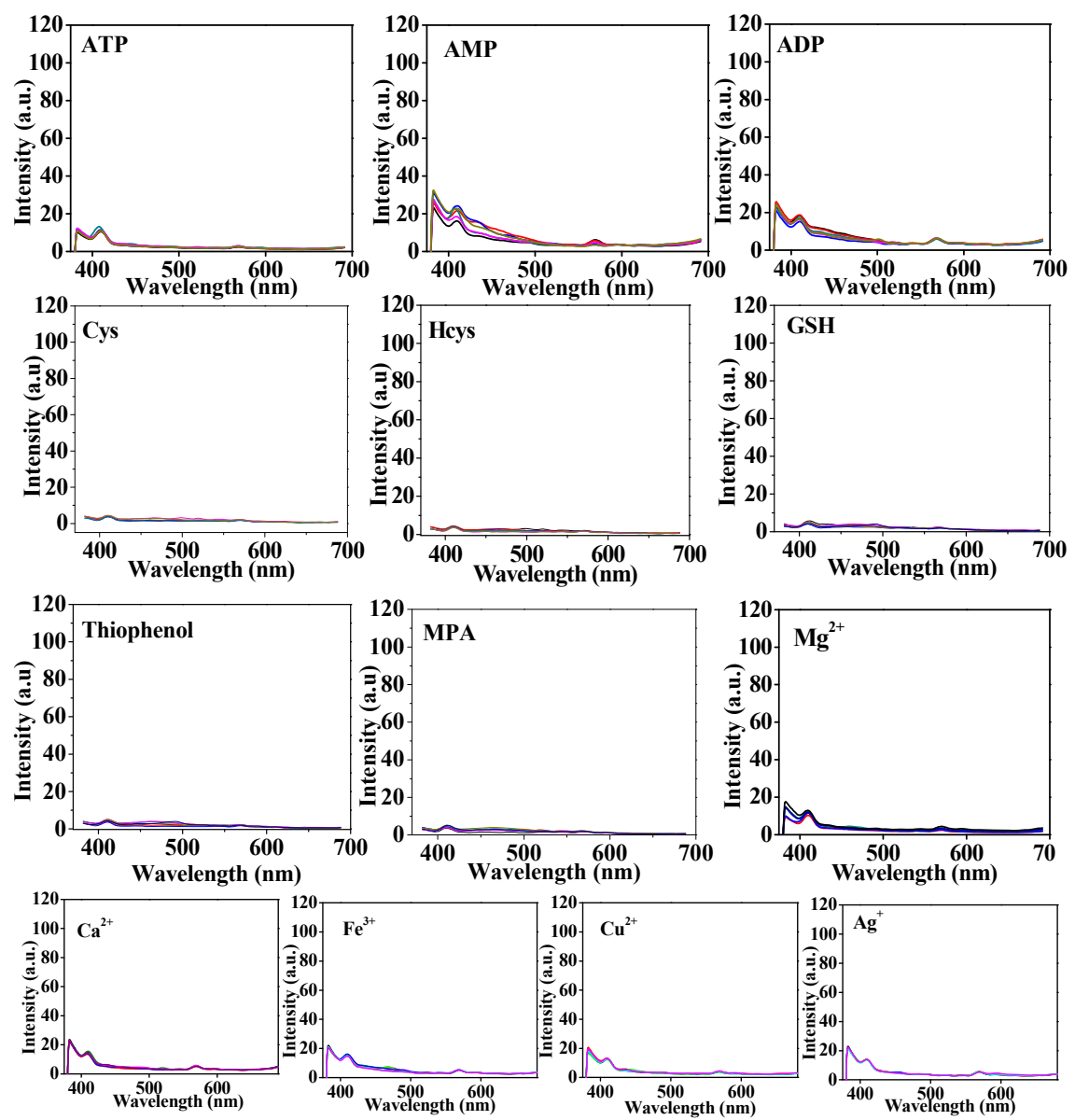

Figure S15: Fluorescence spectra obtained for the titration of ${ }^{5 \mathrm{~F}} \mathbf{L}_{\mathbf{O H}}\left[5 \mu \mathrm{M}, \lambda_{\mathrm{ex}}=360\right.$ $\mathrm{nm}]$ with different anions and metal ion $(100 \mu \mathrm{M})$ in aqueous acetonitrile buffer $\mathrm{pH}$ 7.4.

\section{S16. Determination of Limit of Detection (LOD) of $\mathrm{CN}^{-}$by ${ }^{5 \mathrm{~F}} \mathrm{~L}_{\mathrm{OH}}$}
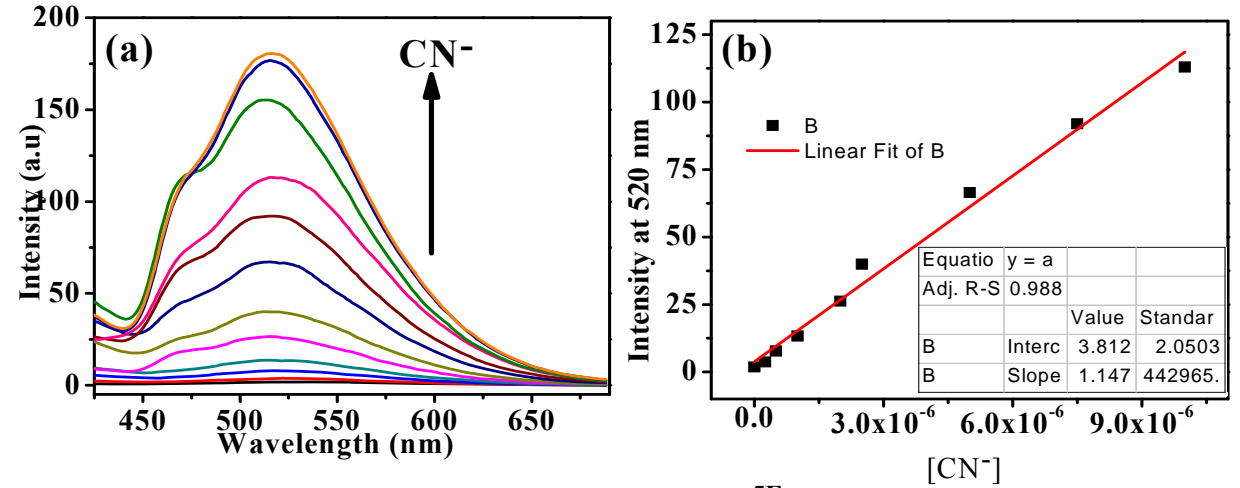

Figure S16. (a) Fluorescence spectral traces of ${ }^{5 \mathrm{~F}} \mathbf{L}_{\mathbf{O H}}$ during titration with $\mathrm{CN}^{-}$to determine LOD. (b) The linear dynamic fluorescence response for the titration of $\mathbf{L}$ with $\mathrm{CN}^{-}$to determine the detection limit (LOD). The LOD was derived by using the formula $3 \sigma / \mathrm{k}$ where $\sigma=$ standard deviation of the blank ( 10 blank samples) and $\mathrm{k}=$ is the slope of linear calibration curve. 
S17. Comparison of the detection limits of recently developed fluorescent probes for $\mathrm{CN}^{-}$in the literature.

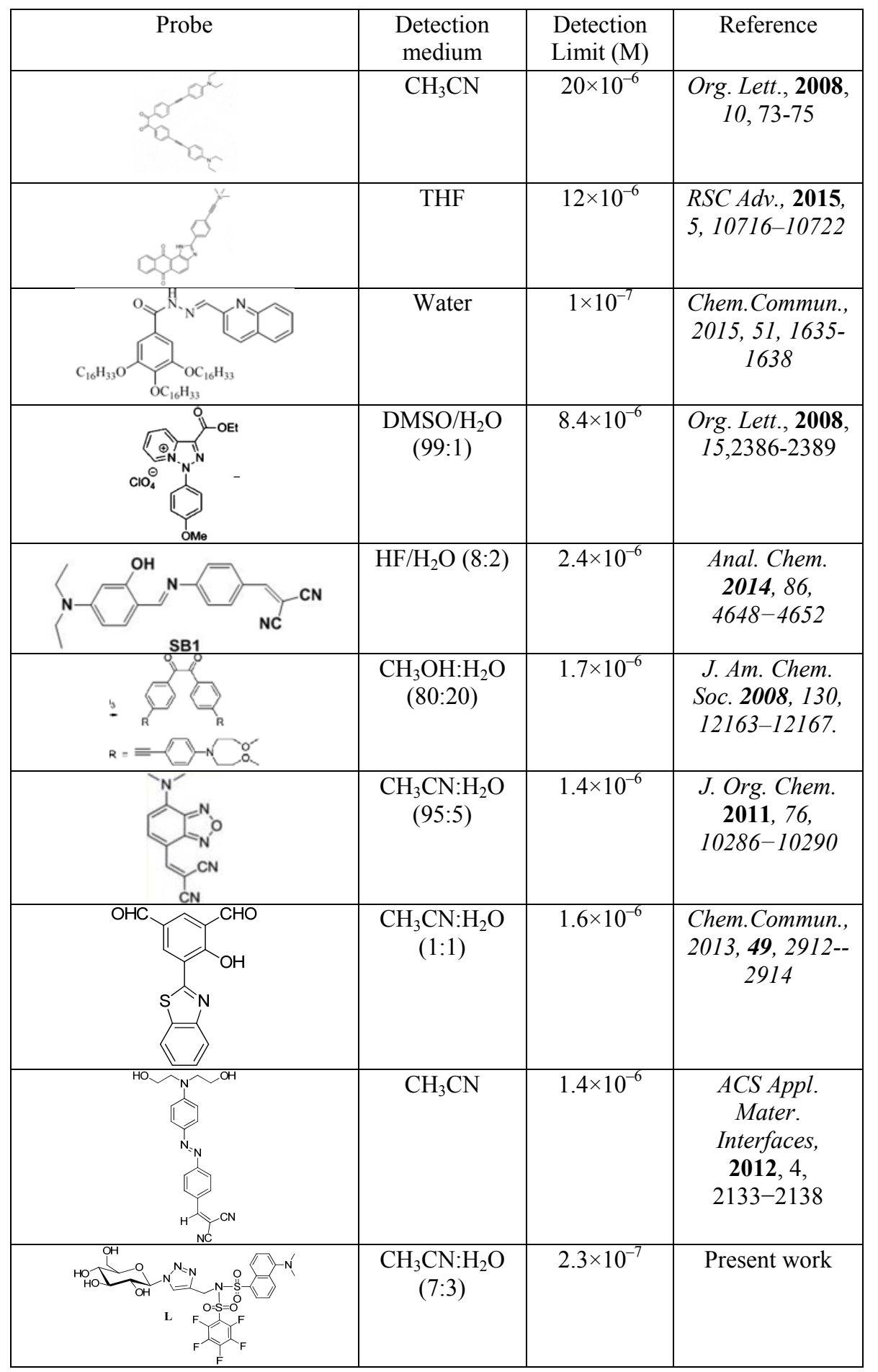


S18. Absorption spectra obtained during the titration $\left[{ }^{55} \mathrm{~L}_{\mathrm{OH}}\right]$ with different anions
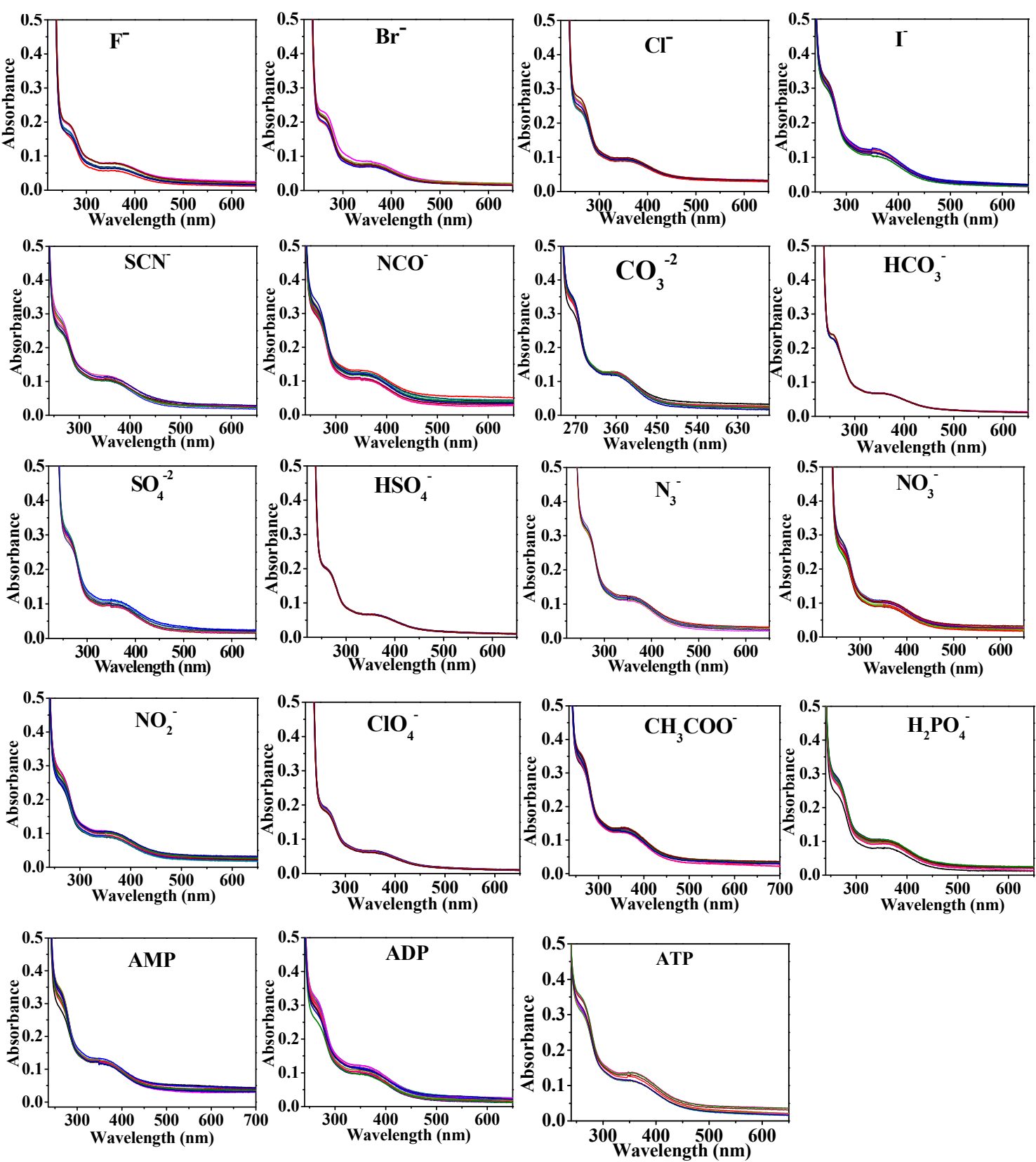

Figure S18. UV-Visible spectral traces obtained during the titration of ${ }^{5 \mathrm{~F}} \mathbf{L}_{\mathbf{O H}}(10 \mu \mathrm{M})$ with different anions $(200 \mu \mathrm{M})$ in aqueous acetonitrile. 
S19. ESI MS spectrum for the titration of $\left[{ }^{5 \mathrm{~F}} \mathrm{~L}_{\mathrm{OH}}\right]$ with $\mathrm{CN}^{-}$
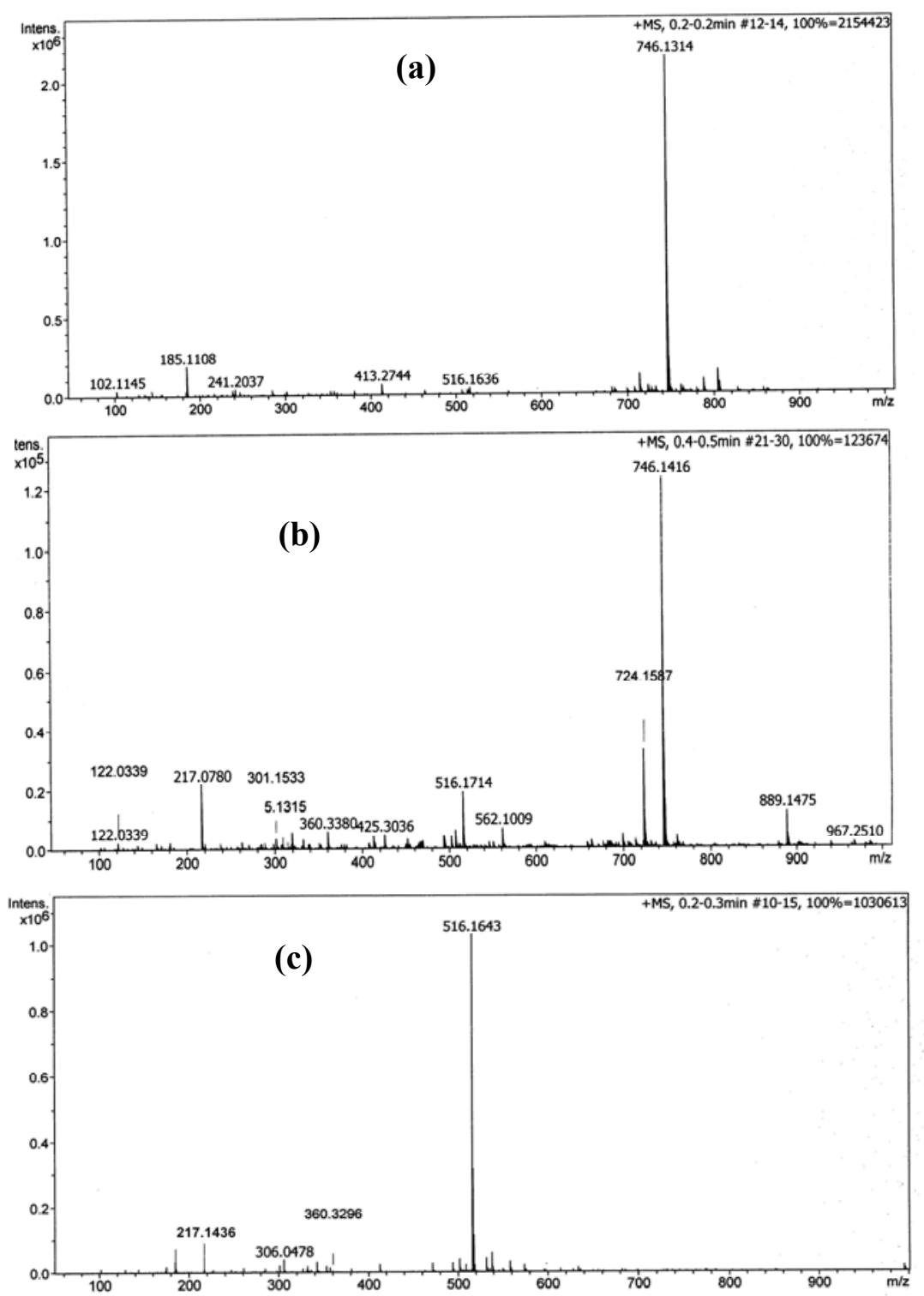

Figure S19. ESI-MS spectral titration of ${ }^{5 \mathrm{~F}} \mathbf{L}_{\mathrm{OH}}$ with $\mathrm{CN}^{-}$: (a) $\left[{ }^{5 \mathrm{~F}} \mathbf{L}_{\mathbf{O H}}\right]$ followed by ' $\mathrm{n}$ ' equivalents of $\mathrm{CN}^{-}$, where, (a) $n=0$, (b) $n=5$, and (c) $n=15$. 
S20. Detection of $\mathrm{CN}^{-}$by ${ }^{5 \mathbf{F}} \mathbf{L}_{\mathbf{O H}}$ on paper strip and silica gel strip.
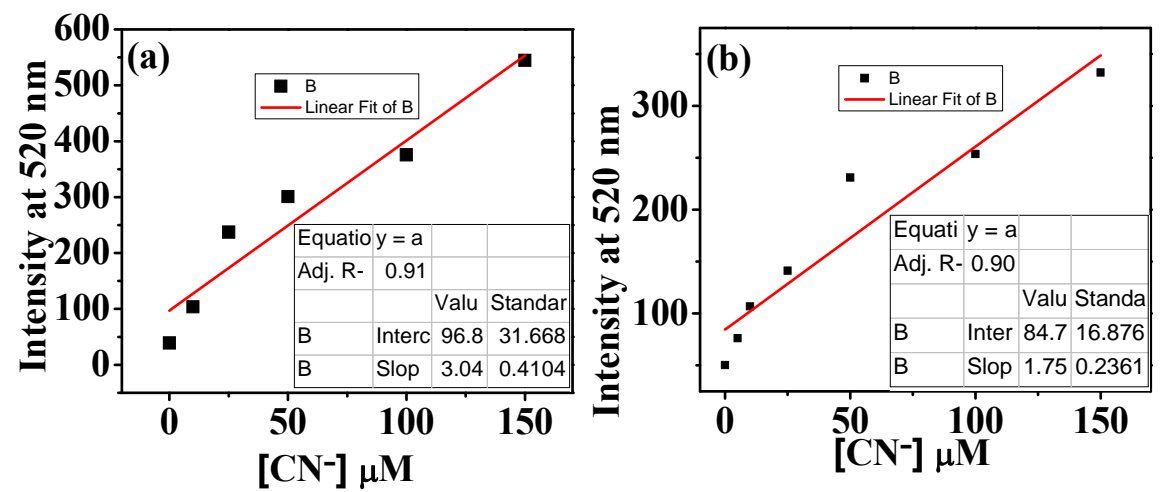

Figure S20. Linear plot obtained in the fluorescence titration of ${ }^{5 \mathrm{~F}} \mathbf{L}_{\mathbf{O H}}$ with $\mathrm{CN}^{-}$in the concentration range 5 to $250 \mu \mathrm{M}$. (a) On the Whatman No. 1 cellulose paper strips, (b) on the silica gel sheets. 
S21. Isothermal titration calorimetry study of ${ }^{\mathrm{nF}} \mathrm{L}_{\mathrm{OH}}$ with $\mathrm{CN}^{-}$
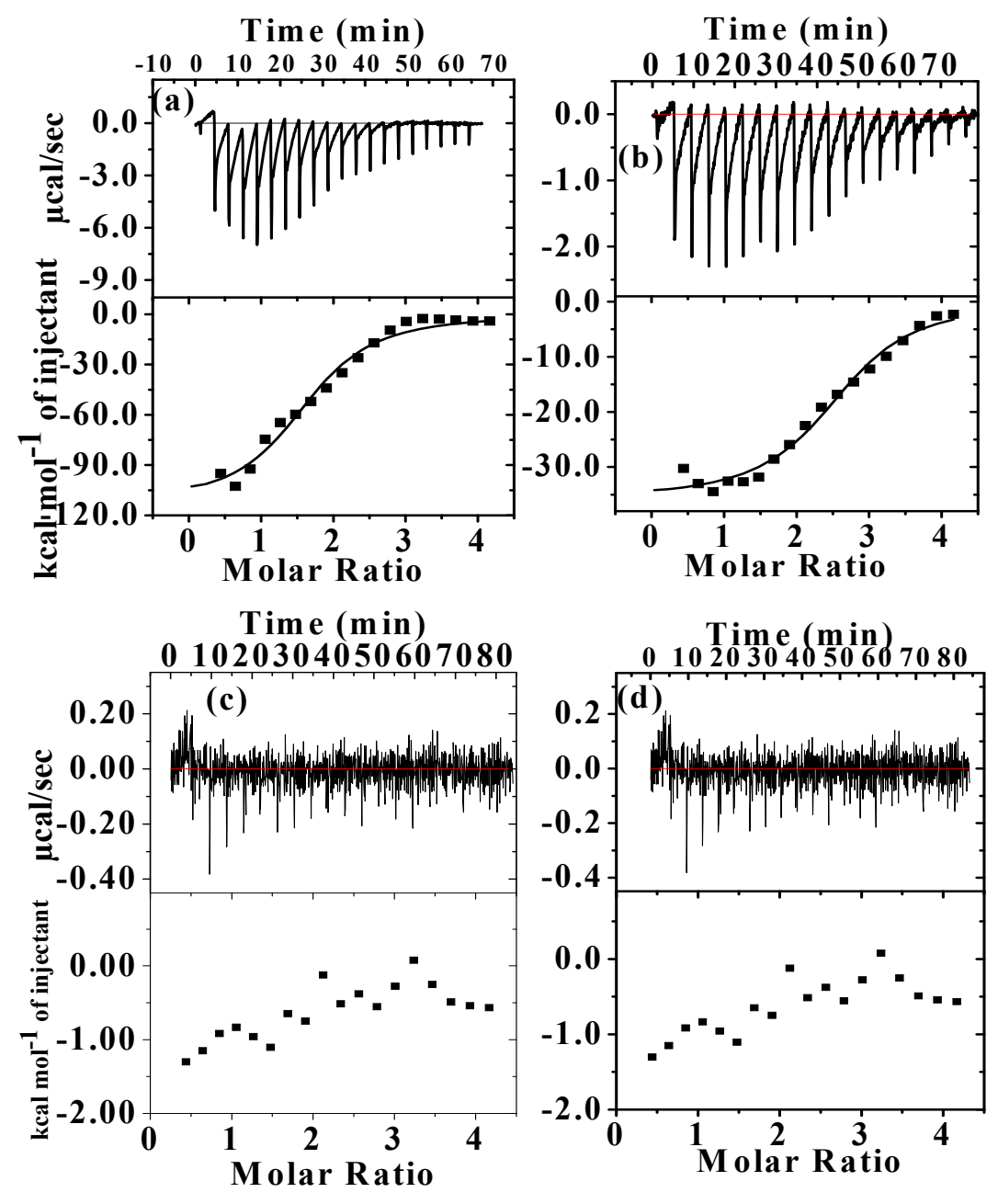

Figure S21. ITC titration of ${ }^{5 \mathrm{~F}} \mathbf{L}_{\mathbf{O H}},{ }^{2 \mathrm{~F}} \mathbf{L}_{\mathbf{O H}},{ }^{1 \mathrm{~F}} \mathbf{L}_{\mathrm{OH}}$ and ${ }^{\mathbf{0 F}} \mathbf{L}_{\mathrm{OH}}(0.1 \mathrm{mM})$ with $\mathrm{CN}^{-}(1 \mathrm{mM})$ in aqueous acetonitrile (V/V, 1:3). ITC thermograms for the reaction of $\mathrm{CN}^{-}$with (a) ${ }^{5 \mathrm{~F}} \mathbf{L}_{\mathbf{O H}}$ (b) ${ }^{2 \mathrm{~F}} \mathbf{L}_{\mathrm{OH}}$ (c) ${ }^{1 \mathrm{~F}} \mathbf{L}_{\mathrm{OH}}$ and (d) ${ }^{\mathrm{OF}} \mathbf{L}_{\mathrm{OH}}$ respectively. Upper panel baseline-corrected raw data for Heats of reaction $v s$ time of $\left[\mathrm{CN}^{-}\right] /[$Probe]. Upper panel heats of reaction $v s$ the mole ratio of $\left[\mathrm{CN}^{-}\right] /[$Probe $]$. The solid line in the lower panel is a best fit obtained upon using the one site model. 


\section{S22. Computational Details}

Density functional theory calculations were carried out using Gaussian 09 [M.J. Frisch, G.W. Trucks, H.B. Schlegel, G.E. Scuseria, M.A. Robb, J.R. Cheeseman, G. Scalmani, V. Barone, B. Mennucci, G.A. Petersson, H. Nakatsuji, M. Caricato, X. Li, H.P. Hratchian, A.F. Izmaylov, J. Bloino, G. Zheng, J.L. Sonnenberg, M. Hada, M. Ehara, K. Toyota, R. Fukuda, J. Hasegawa, M. Ishida, T. Nakajima, Y. Honda, O. Kitao, H. Nakai, T. Vreven, J.A.J.E. Montgomery Peralta Jr., F. Ogliaro, M. Bearpark, J.J. Heyd, E. Brothers, K.N. Kudin, V.N. Staroverov, T. Keith, R. Kobayashi, J. Normand, K. Raghavachari, A. Rendell, J.C. Burant, S.S. Iyengar, J. Tomasi, M. Cossi, N. Rega, J.M. Millam, M. Klene, J.E. Knox, J.B. Cross, V. Bakken, C. Adamo, J. Jaramillo, R. Gomperts, R.E. Stratmann, O. Yazyev, A.J. Austin, R. Cammi, C. Pomelli, J.W. Ochterski, R.L. Martin, K. Morokuma, V.G. Zakrzewski, G.A. Voth, P. Salvador, J.J. Dannenberg, S. Dapprich, A.D. Daniels, O. Farkas, J.B. Foresman, J.V. Ortiz, J. Cioslowski, D.J. Fox, Gaussian 09, Revision D.01, Gaussian, Inc., Wallingford, CT (2010)].in order to establish the reaction coordinates profile of $-\mathrm{CN}^{-}$towards ${ }^{5 \mathbf{F}} \mathbf{L}_{\mathbf{O H}}$. In this study, we assess the performance of four different DFT functionals, i.e., a hybrid functional (B3LYP), functionals including dispersion i.e $\mathrm{B} 97 \mathrm{D}$ and $\mathrm{wB} 97 \mathrm{xD}$.

\begin{tabular}{|c|c|c|c|c|}
\hline Parameter & & \multicolumn{3}{|c|}{ Level of theory } \\
\hline & X-ray & B97D & wB97xD & B3LYP \\
\hline r1 & 3.257 & 3.196 & 3.274 & 3.847 \\
\hline r2 & 1.706 & 1.779 & 1.714 & 1.756 \\
\hline r3 & 1.649 & 1.745 & 1.692 & 1.708 \\
\hline r4 & 1.503 & 1.505 & 1.488 & 1.499 \\
\hline r5 & 1.502 & 1.599 & 1.494 & 1.498 \\
\hline r6 & 1.438 & 1.469 & 1.456 & 1.462 \\
\hline a1 & 120.2 & 119.2 & 121.0 & 121.2 \\
\hline $\mathrm{a} 2$ & 113.1 & 112.9 & 113.2 & 115.8 \\
\hline
\end{tabular}


Figure S22: X-ray crystal geometries along with ${ }^{5 \mathrm{~F}} \mathbf{L}_{\mathbf{O H}}$ optimized geometries have a given at four different level of theory (Geometries are given in $\AA$ and Angels are given in degree).

The bond lengths and bond angles were compared to available X-ray crystal parameters of ${ }^{5 \mathrm{~F}} \mathbf{L}_{\mathbf{O H}}$ at four different levels of theory. For structure ${ }^{5 \mathrm{~F}} \mathbf{L}_{\mathbf{O H}}, \mathrm{r} 1, \mathrm{r} 2$ and $\mathrm{r} 3$ distances of 3.274 $\AA, 1.714 \AA$ and $1.692 \AA$ were calculated, which compare well with the corresponding experimental values, $3.257 \AA, 1.706 \AA$ and $1.649 \AA$ respectively. Bond angles were also reproduced accurately, apart from "a1" $(\angle \mathrm{S}-\mathrm{N}-\mathrm{S})$, which is slightly overestimated by ca. $1^{\circ}$. Among these three levels of theory, wB97xD yields structural parameters which are close to those from X-ray data. The B97D and B3LYP functionals slightly over-estimate bond lengths and at B97D under-estimate the bond angles somewhat. Following the good performance of $\mathrm{wB}^{\mathrm{B}} 97 \mathrm{xD}$ for prediction of geometrical properties, we have utilised this level of theory for the remaining calculations, and results have been analyzed, explained and rationalized at this level of theory.

The exited state calculations were carried out with TDDFT using all four levels on the ground state (TDDFT//DFT optimized structures) optimized geometries and results are shown in Table S22.

Table S22: Selected electronic excitation energies $(\mathrm{eV})$ and oscillator strengths $(f)$, configurations of the low-lying excited states of the ${ }^{5 \mathrm{~F}} \mathbf{L}_{\mathbf{O H}}$ and the product structure, calculated by TD-DFT//DFT/6-31G(d,p), based on the optimized ground state geometries.

\begin{tabular}{rcccrl}
\hline \multicolumn{5}{c}{ TDDFT//wB97xD/6-31G(d, p) } \\
\hline \multirow{2}{*}{ Structure } & $\begin{array}{l}\text { Electronic } \\
\text { transitions }\end{array}$ & Excitation energy & $\mathrm{f}^{\mathrm{b}}$ & Composition $^{c}$ & $\mathrm{CI}^{d}$ \\
\hline${ }^{\mathbf{5 F}} \mathbf{L}_{\mathbf{O H}}$ & $\mathrm{S}_{0^{---}>\mathrm{S}_{1}}$ & $3.9581 \mathrm{eV}(313.24 \mathrm{~nm})$ & $\mathrm{f}=0.1439$ & $\mathrm{H}--->\mathrm{L}$ & 0.69349 \\
$\mathbf{L}_{\mathbf{1}}$ & $\mathrm{S}_{0^{---}>\mathrm{S}_{1}}$ & $4.0737(304 \mathrm{~nm})$ & $\mathrm{f}=0.1919$ & $\mathrm{H}--->\mathrm{L}$ & 0.69324 \\
\hline
\end{tabular}

$a$ The numbers in parentheses are the excitation energy in wavelength. $b$ Oscillator strength. $c$ H stands for HOMO and L stands for LUMO. Only the main configurations are presented. $d$ Coefficient of the wave function for each excitation. The CI coefficients are in absolute values 